University of Louisville

ThinkIR: The University of Louisville's Institutional Repository

Electronic Theses and Dissertations

8-2006

\title{
Multiobjective simulation-based methodologies for medical decision making.
}

Ahmed Hassan YoussefAgha

University of Louisville

Follow this and additional works at: https://ir.library.louisville.edu/etd

\section{Recommended Citation}

YoussefAgha, Ahmed Hassan, "Multiobjective simulation-based methodologies for medical decision making." (2006). Electronic Theses and Dissertations. Paper 1627.

https://doi.org/10.18297/etd/1627

This Doctoral Dissertation is brought to you for free and open access by ThinkIR: The University of Louisville's Institutional Repository. It has been accepted for inclusion in Electronic Theses and Dissertations by an authorized administrator of ThinkIR: The University of Louisville's Institutional Repository. This title appears here courtesy of the author, who has retained all other copyrights. For more information, please contact thinkir@louisville.edu. 


\title{
MULTIOBJECTIVE SIMULATION-BASED METHODOLOGIES FOR MEDICAL DECISION MAKING
}

\author{
By \\ Ahmed Hassan YoussefAgha \\ B.Sc., Menofia University, Egypt, 1985 \\ MSCS., University of Louisville, KY, US, 2001 \\ MSPH., University of Louisville, KY, US, 2003
}

\author{
A Dissertation \\ Submitted to the Faculty of the \\ Graduate School of the University of Louisville \\ In Partial Fulfillment of the Requirements \\ for the Degree of
}

Doctor of Philosophy

Department of Biostatistics and Bioinformatics

University of Louisville

Louisville, Kentucky

August, 2006 


\title{
MULTIOBJECTIVE SIMULATION-BASED METHODOLOGIES FOR MEDICAL DECISION MAKING
}

\author{
By \\ Ahmed Hassan YoussefAgha \\ B.Sc., Menofia University, Egypt, 1985 \\ MSCS., University of Louisville, KY, US, 2001 \\ MSPH., University of Louisville, KY, US, 2003
}

A Dissertation Approved on

June 21, 2006

By the following Dissertation Committee

Gerald W. Evans, Ph.D.

Dissertation Director

Steven McCabe, MD, MSc.

Adel S. Elmaghraby, PhD.

John Myers, PhD.

Scott LaJoie, PhD. 


\section{DEDICATION}

This dissertation is dedicated to my Parents

Mr. Hassan YoussefAgha

And

Mrs. Fawzia Youniss

who have given me invaluable educational opportunities. 


\section{ACKNOWLEDGMENTS}

After thanks to ALLAH (GOD), I would like to thank my committee members: Prof. Gerald W. Evans, Dr. Steven McCabe, Prof. Adel Elmaghraby, Dr. John Myers, and Dr. Andrew Scott LaJoie. In particular, I am so appreciated to my mentor Prof. Gerald W. Evans for his superior supervision and continuous help and follow up of every point in my research. Also, I would like to thank very much Dr. Steven McCabe for his real support and assistance all the time. Indeed, I can not express my respect to Prof. Adel S. Elmaghraby, who had recommended to my interest to study at the Biostatistics-Decision Sciences Program, at the School of Public Health, the UofL.

Many thanks go to my father Mr. Hassan YoussefAgha, my mother Mrs. Fawzia Youniss, and my wife Ghada, who prayed for me; who encouraged me through their unconditional love and continuous support to finish my $\mathrm{PhD}$. I hope that my handsome son Zeyad to be a doctor of aerospace engineering as he wishes, and I hope that my lovely and gorgeous daughter Hanna to be a great mother and a famous scientist or MD. I have to mention my friend and brother Mohamed Hussein for his valuable suggestions.

Again,

Thanks to ALLAH

Ahmed YoussefAgha 


\title{
ABSTRACT \\ MULTIOBJECTIVE SIMULATION-BASED METHODOLOGIES FOR MEDICAL DECISION MAKING
}

\author{
By \\ Ahmed Hassan YoussefAgha
}

June 21,2006

A variety of methodologies have been employed for decision making related to the treatment of diseases/injury. Decision trees are a functional way in which to examine problems under uncertainty by providing a method to analyze decisions under risk (Detsky, 1996, 97). However, conventional decision trees do not completely represent "the real world" since they cannot investigate problems that are cyclic in nature (Jaafari, 2003).

The stochastic tree that developed Hazen during 1992-to-1996 is one of the most relevant methods and techniques related to decision analyses that append more incorporation for medical intervention related to recurring diseases/injuries. "The approach combines features of continuous-time Markov chains with those of decision trees and that enable time to be modeled as a range where health state transitions can occur at any instant" (Hazen 1992-to-96). It can also accommodate patients' preferences regarding risk and quality of life.

The importance of Hazen's stochastic tree was mentioned in the technical report, by Prof. Keefer et al, Arizona State University, Summary of Decision Analysis 
Applications in the Operations Research Literature on 1990-to-2001 (Keefer, 2002).

They stated that the work of Hazen, stochastic trees, is one of applications that presented significant advancement in decision analysis methodological tools.

However, in medical decision making, Hazen stochastic tree model has some limits and restrictions. Hazen stochastic tree can only utilize an exponential distribution for health state sojourn, to simplify the rollback/recursive computations. Noticeably, some diseases/injury can be best represented by distributions such as Weibull distribution, Gamma distribution, Log-Logistic distribution, Log-Normal distribution, and Coxian distribution (Collett, 1999). In addition, the stochastic tree modeling technique does not have a method to correct differences between experimental circumstances/conditions and the corresponding circumstances/conditions of a standard/current treatment.

In this research we enhance Hazen's stochastic tree by developing an analytical model, and we extend its capabilities more by developing multi-objective simulation based methodologists for medical decision making. First, with our enhancement on the Hazen's stochastic tree, the model is improved by utilizing the Weibull Accelerated Failure Time model. This new technique will fill the gap between the experimental circumstances and the corresponding circumstances or conditions of standard/current treatment. Second, as simulation can be a final alternative for problems that are mathematically intractable for other techniques (Banks 1996), our multi-objective simulation based model for medical decision making extends the capabilities of Hazen stochastic tree. It adds more flexibility with the use of survival distributions for health 
states sojourn, and combines two sound theories: multi attribute utility (MAU) theory, and Ranking-Selection procedures.

Indeed, our simulation model (considering patient's profile/preferences and health states survival/quality/cost, QALY) presents an investigation of the use of simulation on the stochastic tree, with associated techniques related to ranking and selection, and multiobjectives decision analysis.

Key words: Stochastic Tree, Treatments Models, Simulation Methodologies, Multi --objectives Decision Analysis, Patient's Profile, Patient's Preferences, Quality Adjusted Life Year (QALY), Health States Cost. 


\section{TABLE OF CONTENTS}

\section{PAGE}

ACKNOWLEDGMENT ..................................................

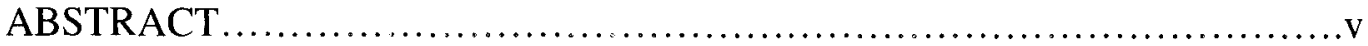

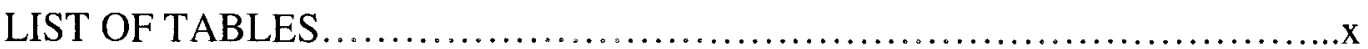

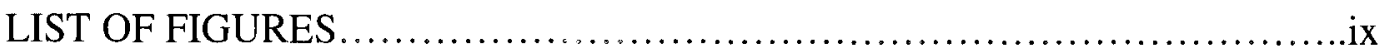

\section{CHAPTER}

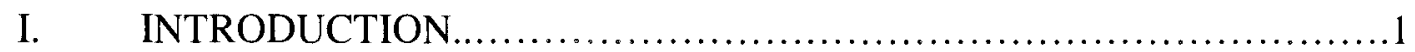

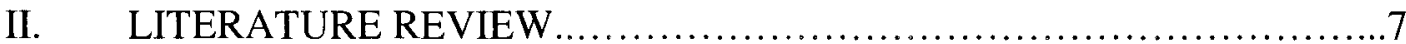

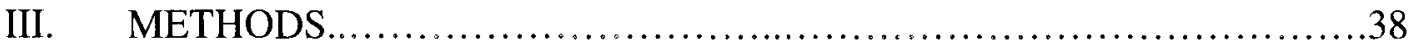

An enhancement of the stochastic tree model for medical decision making..38

Multiobjective simulation-based methodologies for medical decision making on recurrent diseases treatment......................................................55

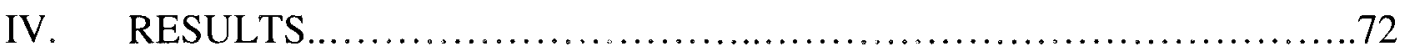

Example I on the Enhanced Stochastic Tree Model on Stroke .......76

Example II on the Simulation Model of the Stochastic Tree Model on Three Breast Cancer Treatments.................................... 79

Sensitivity Analysis...........................................86

V. DISCUSSION AND CONCLUSION ................................... 87

Our Approach Advantages.........................................89

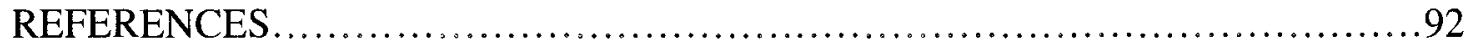

CURRICULUM VITAE...................................................97 


\section{LIST of TABLES}

TABLES

PAGE

1. Pilot experiment on Ranking \& Selection 31

2. Pilot experiment on Ranking \& Selection Result 31

3. A Pilot Experiment on MCP 33

4. Final Result on a Pilot Experiment on MCP 33

5. Comparison Methods' Differences and Similarities 34

6. Comparison Methods Advantages and Disadvantages 34

7. Results of Stochastic Tree Model to Compute QALY for Stroke 77

8. Quality of Life (QoL) 80

9. Health State's Stay Time Distributions, in years 80

10. Annual treatment Cost $\quad 80$

11. Initial Values for the simulation system 80

12. Transition Probability Matrix $\quad 81$

13. Transition Rates 81

14. CDIS pilot experiment; the first stage results 84

15. CDIS the second stage results $\quad 84$

16. CDIS pilot experiment for the sensitivity analysis; the first stage results 85

17. CDIS the second stage results, for the sensitivity analysis 85 


\section{LIST OF FIGURES}

FIGURE

PAGE

1. Three different curves of Weibull Density function 42

2. Our General Simulation Model's Components 45

3. Risk avoider utility function on performance measure, QALY 60

4. Risk avoider utility function on performance measure, Treatment Cost 61

5. Continuous-risk assessment approach, by Hazen 1995

6. Our General Simulation Model, on Arena 64

7. Our General Simulation Model Initials 65

8. Our General Simulation Model Transition Probabilities settings 65

9. Our General Simulation Model Stay time Assignments 66

10. Our General Simulation Model Performance Measures Assignments 66

11. Our General Simulation Model Performance Measures Recording 67

12. Our General Simulation Model Advancing Time 67

$\begin{array}{ll}\text { 13. Indifference-zone and preference-zone diagram } & 71\end{array}$

14. Schematic overview of Stroke Outcome Model. 75

15. Stochastic Tree Model to Compute QALY for Stroke 77

16. The Simulation model for Mastectomy 82

17. The Simulation model for Lumpectomy Only 82

18. The Simulation model for Lumpectomy followed by Radiation therapy 82 


\section{CHAPTER I}

\section{INTRODUCTION}

Typical decision trees are a functional way in which to analyze problems under uncertainty by providing a mechanism to analyze decisions under risk (Detsky 1996, 97). Conventional decision trees examine complex issues composed of discrete sequential events such as, undergoing tests, receiving the tests results, and deciding on the optimal medical management. Conventional decision trees do not completely represent "the real world" since they cannot investigate disorders that are cyclic in nature (Jaafari, 2003). As a result, researchers have developed methodologies, via Markov processes, allowing for the examination of cyclic problems or recurrent diseases.

Moreover, in medical decision making, decision trees models have some limits and restrictions, such as having a low dimensional preference summary (e.g. memory less, Markov, or Semi-Markov) to allow or feasibility for rollback calculations, lack of filling the gap between the experimental and practical medical intervention, limits related to specific survival distributions, and no capabilities to unify multiple performance measures into one scalar. So, further work is required to add more capabilities and extensions onto decision tree models to examine/study, accurately and with more reality, the health outcomes of medical interventions associated with cyclic disorders or recurrent diseases. 
Simulation is "a broad collection of methods and applications to mimic the behavior of real systems, usually on a computer with suitable software" (Kelton, 2001). Simulation is a methodology that has advantages, such as: (a) simulation can be a solution methodology of last alternative for problems that are mathematically intractable for other techniques; (b) even for problems that are mathematically tractable, simulation can often provide a higher level of detail than can other techniques. (c) Simulation can sometimes provide (approximate) answers at a smaller cost/effort to some problems which are fully tractable mathematically but whose solution may be cumbersome and time consuming (Banks 1996). Other advantages of simulation include the facts that it permits modification or design of medical intervention systems by trial and error, allows for easy exploration of the system's sensitivity to changes in the input parameters, and provides a highly controllable environment for experiments. Simulation can be valuable to the operations researcher as a means of testing the applicability and validity of mathematical models and expressions.

This study has two main parts: First, a special case analytical model is developed, based on the earlier work of Hazen (1992-to-1996). Second, a simulation-based model is developed for further study.

The stochastic tree model developed by Hazen combines features of continuoustime Markov chains with those of conventional decision trees to analyze cyclic disorders and/or recurrent diseases/injuries (Hazen, 1992-1996).

Hazen's stochastic tree model allows health state transitions to occur at any instant. Hazen's work, of stochastic tree, presents significant detail about a particular decision analysis methodological and implementation issue: in particular Hazen 
described and presented the development and the use of a decision tree model; moreover, it discussed in depth subjective utility/value functions and tradeoffs between attributes. Hazen's stochastic tree technique resolved problems, related to recurrent diseases and injuries, inherent in conventional decision analysis methodologies (Keefer, 2002).

The Hazen et al. model (Hazen, 1992-1996) maintains multiple input parameters and one performance measure, at a time; and run within constraints for recursive processing. In stochastic tree technique, the time spent in any health state is restricted to a low-dimensional or tractable preference summary (e.g. memory less, Markov, and SemiMarkov) which guarantees both tractability recursive computations and convenience in assessment. Hazen's stochastic tree mode can only utilize an exponential distribution for health states stay time. Noticeably, some disorders can be best represented by different distributions such as Weibull distribution, Gamma distribution, Log-Logistic distribution, Log-Normal distribution, Coxian, and Quadratic distribution (Collett, 1999). In addition, the stochastic tree modeling technique does not have a method to correct differences between experimental circumstances/conditions and the corresponding circumstances and/or conditions of the standard/current treatment.

A performance measure such as quality-adjusted life years (QALY) is the measure of health benefit to an individual. QALY reflects the gain in expected utility of having some treatment. It can be used to represent utility only if individuals are willing to trade off years of life in a given health state for fewer years at an ideal health state at a constant rate, irrespective of the number of years spent in the state (Clarke 2004). In addition, it would be beneficial if these methodologies integrate an individual's specific health conditions such as smoking history, body mass index, and age. 
For the first part of this research, we extend Hazen's stochastic modeling technique, which incorporates the memory-less exponential distribution by utilizing the Weibull Accelerated Failure Time model (WAFT). The WAFT model is memory-less when Weibull's shape parameter equals to one. This new technique includes health conditions at the individual level and may not only provide a novel way in which to study QALY or other medical intervention outcomes, but also it may provide a novel way in which to analyze recurrent diseases. However, the enhanced model is still restricted to have a particular tractable preference summary- represented in the use of WAFT with shape parameter equals to one to fit with the memory less property the same as the use of the exponential distribution- for facilitating rollback computations. Consequently, it was still limited to the use of specific survival distributions, and had no capabilities to unify multi-performance measures into one scalar at a time. So, further work was required to add more capabilities and extensions not only for the related limitations on the use of specific survival distribution, but also to add multiple performance measures.

Most of the ranking and selection $(\mathrm{R} \& \mathrm{~S})$ literature focuses on procedures that are designed for scalar performance measures (Bechhofer, 1995). However, some multivariate results do exist: Gupta (1979) described procedures that are based on scalar functions of the mean and covariance matrix of the multivariate populations. These procedures reduce the multivariate performance measure problem to a scalar performance measure problem. The procedures described in Gupta, require a complicated step of estimating a covariance matrix, and the approach does not belong to a class of procedures whose properties are easy understood (Morrice 2001). Kim et al utilized a Maxi-Min approach to optimize the characteristics of steel (Kim and Lin, 1999). Kim indicated, in 
some situations that a Maxi-Min method may cause an unreasonable decision because it focuses on maximizing the poorest performing measure. For this reason, they advised performing "several approaches for the final decision" (Kim 1999, page 8).

"In a medical related business setting, a different approach is often used: convert project performance over multiple measures to a scalar measure using costs" (Morrice 2001, page 800). Even though this "costing" method of performance measures has advantages, it has some disadvantages too. Not enough resources and not accurately assigning a dollar value to intangibles and ambiguous measures (e.g., quality of life) affect perfect cost even though the data resource available.

In our simulation modeling, we utilize an approach, similar to Morrice et al. (Morrice 1998, 1999), to this problem: convert multiple performance measures to a scalar performance measure using multiple attribute utility (MAU) theory (Keeney and Raiffa 1992). MAU theory can be used with or without costing approach when good cost data are not available or when cost is not suitable as a measure of performance.

For the second part of this research, we extend Hazen's stochastic tree using simulation model, MAU theory, and a statistical R\&S with the indifference-zone approach of (see Law and Kelton, 2000), to select the best medical intervention configuration from a set of $n$ configurations when a medical intervention performance is determined by $m>1$ performance measures. In other words, we developed a simulation model that maintains multiple input parameters and multiple performance measures.

Chapter2-Literature Review contains seven main sections: Decision Tree and Stochastic Tree, Markov Models, Survival Analyses, Screening and Ranking-Selection Procedures, Multiple Attribute Utility Theory, and Simulation. Each section defines, 
describes, and illustrates the advantages and the disadvantages of the related decision model. Chapter3-Methods is divided into two parts. For the first part, four main sections explain the purpose of enhancing Hazen's stochastic tree model; then it goes through the enhanced model specifications, model updating using Weibull accelerated failure time model, and ends with a conclusion section. In the second part of the chapter, nine main sections describe our design of the simulation model that extends the use of the stochastic tree, details of how one uses the simulator in conjunction with MAU theory, the application of statistical ranking and selection, $R \& S$, procedures to select the best medical intervention configuration of multiple possible configurations. Chapter4-Resultsillustrates examples for the application of the analytical and the simulation models, and discusses sensitivity analysis on models outputs; and finally it displays the advantages of our models. 


\section{CHAPTER II}

\section{LITERATURE REVIEW}

\subsection{BACKGROUND}

Reviewing the most relevant methods and techniques related to decision analyses that can integrate or that append more integration for medical intervention in recurring diseases/injuries/disorders would be beneficial for the person who is interested in medical decision making application.

Hazen's stochastic tree model based on medical decision tree methodology with a technique for solving continuous-time Markov cycle trees. But, the model was restricted to the exponential distribution for health states stay. So, in this chapter, section 2 to 4, we discuss and explain: decision-stochastic trees and their limits in section (2), Markov models and their limits in section (3), and survival analysis and its limits in section (4). In sections 5 to 7, we discuss and explain: Ranking-Selection procedures in section (5), Multi attribute utility theory in section (6), and finally will focus, in section (7), on Simulation models and simulation output analysis. 


\subsection{DECISION TREE - AND- STOCHASTIC TREE MODELS}

2.2.1 Decision Tree: Moore defined a decision tree by: it is a graphical device for analyzing decision under risk (Moore, 2001). A decision tree is an essential element of decision analysis under uncertainty (Raiffa, 1968; and Jaafari, 2003). One of the advantages of decision trees is the ability it offers to structure the decision problem by mapping out all feasible alternative actions. As such, it is mainly useful for analyzing complex sequential decisions when uncertainty is resolved at separate, discrete points in time.

2.2.1.1 Decision Tree Limits: a decision tree is not a complete representation of "the real world" but rather a simplified and highly stylized model of the most important components (Allan Detsky, 1997). Decision analysis by traditional decision tree might be misused by decision makers tending to focus only on the initial decision to accept or reject the intervention at the cost of subsequent decisions being dependent on it, decision tree analysis forces decision maker to bring to the interdependences between the initial decision and subsequent decisions" (Jaafari, 2003). "With events and sequential decisionmaking over extended periods of time, where consequences cannot be defined to cover the future, in a medical context, a Markov process had been incorporated into a decision tree to overcome the unmanageable decision tree" (Pliskin 1975). Also, to handle a continuous nature, there are two main options to handle such a situation: (1) approximate the continuous outcomes with a Pearson-Tukey approach, or (2) use the technique of Monte Carlo simulation designed to handle probability distributions of a continuous nature. (Moore, 2001) 
2.2.2 Stochastic Tree: For enhancing the medical decision methodology, Beck and Pauker (1983) extended medical decision tree methodology to consider discrete-time Markov process-based models; which is helpful when the timing of a treatment is a critical variable. Recently, Hazen $(1992,93,96,98, \& 2002)$ has developed stochastic trees as a technique for solving continuous-time Markov cycle trees.

Hazen et al, 1992-to-2002, in the medical decision making field, has developed an approach that combines features of continuous-time Markov chains with those of decision trees and that enable time to be modeled as a range where health state transitions can occur at any instant. "It can also accommodate patients' preferences regarding risk and quality of life. The stochastic tree concerns itself with the recursive evaluation of utility function, that it is the calculation of an expected utility measure using iterative methods (CYCLIC) similar to the method of successive approximations (value iteration) in the stochastic Dynamic Programming”.

2.2.2.1 Stochastic Tree Limits: the stay in any health state is restricted to lowdimensional preference summary (e.g. memory less) for the recursive computations and to fit with the Markov process property. So, Hazen's stochastic tree model was created with respect to exponential distribution only; nevertheless, there are other survival data should be represented in different sojourn (survival models) distributions, such as Weibull distribution, Gamma distribution, Log-Logistic distribution, Log-Normal distribution, Coxian, Quadratic, and Bathtub distribution that could be used (Collett, 1999). 


\subsection{MARKOV MODELS}

Markov models provide a solution to the problem of modeling the natural history of chronic disease. Their simplicity, computational ease, and broad applicability to many clinical- and health-related situations make Markov models more attractive than other more complicated, modes of analysis. The Markov model assumes that the future is independent of the past given the present. When using Markov the random variable is indexed in time, which can be either discrete or continuous. Many random events are affected by what has happened before.

Decision trees often include Markov models to calculate final outcomes or simply to collapse a bushy branch into one that is more manageable. The Markov model simplifies decision trees by defining a series of transition probabilities between health states. These probabilities define the progress of patients through the model.

2.3.1 Classes of Markov Models: There are two broad classes of Markov models, Markov chains \& Markov processes, defined by the determinants of the state transition probabilities. Markov chains contain constant transition probabilities, whereas Markov processes have time-dependent transition probabilities. Markov chains are easy to use and compute in cases where the disease has a short time horizon, because of the probability restriction; however, with more chronic conditions, the chance of moving between health states increases with age. Aging increases the risk of transition from a healthy state to one of sickness, thereby violating the constant state-transition probability assumption of Markov chains. Markov processes are used to model disease over longer time periods. 
Behaving similar to the pure Markov model there is the semi-Markov process

model. It is a probabilistic model useful in analyzing complex dynamical systems.

However, with semi-Markov models, the transition rates in a particular state depend on the time already spent in that state (sojourn time) but that they do not depend on the path by which the present state was reached. Thus transition distributions in the semi-Markov process can be non-exponential. The semi-Markov theory involves the concepts of state and state transition. The most important statistics of the semi-Markov process are the interval transition probabilities.

Markov processes are useful in representing events where the risk or frequency of events varies over time. Markov models and Markov chain theory have been applied to a variety of areas in science and medicine (Hazen literature group, 1992).

2.3.1.1 Markov Models Limits: While conceptually and graphically welldesigned for representing event probabilities with repetitive chances, Markov models inherently are limited by the Markov property restriction and other assumptions placed on the transition probabilities. Recall that in the Markov model, the current state of health is the only information used to predict state transition probabilities. The Markov model has no memory of past events. This assumption often is violated in clinical situations, where most clinicians use past information to guide treatment decision making.

2.3.3 Markov Decision Process: a Markov Decision Process (MDP) is just like a Markov Chain, except the transition matrix depends on the action taken by the decision maker (agent) at each time step. The agent receives a reward, which depends on the action and the state. The goal is to find a function, called a policy, which specifies which action to take in each state, so as to maximize some function of the sequence of rewards. 
One can formalize this in terms of Bellman's equation, which can be solved using policy iteration. (Feinberg, 2002)

Markov decision process is a mathematical representation of a sequential decision making problem in which: A system evolves through time. A decision maker controls it by taking actions at pre-specified points of time. Actions acquire immediate costs or accumulate immediate rewards and affect the subsequent system state. A MDP is used to identify a policy that maximizes the expected utility/reward.

2.3.3.1 Markov Decision Process limits: the theory of MDP studies sequential optimization of discrete time stochastic systems. The basic objective of MDP is a discrete-time stochastic system whose transition mechanism can be controlled over time. The concept of dynamic programming, which is very important for MDPs, was systematically studied by Bellman in many publications; this concept has been used by several authors to approach various problems (see Feinberg, 2002).

The dynamic programming principle in its classical form can be applied only to problems with an appropriate single objective function. For some objective functions or when the goal is to optimize one objective function under constraints on other criteria, the problem usually cannot be solved directly by dynamic programming. Convex analysis methods (Eugene, 2002, chapter 11), including linear and convex programming in finite and infinite dimensional spaces are usually more natural in these situations. 


\subsection{SURVIVAL ANALYSES}

Disease progression and its effect on survival follow a distribution related to age. More generally, systems or events that "decay" over time can be modeled using a survival or hazard function (Collett, 1999). Survival analysis and the hazard function are useful methods to model treatment effectiveness and program costs. The hazard function relates survival probabilities to survival times, to treatment costs, or to any other related factor.

In clinical trials, treatment efficacy often is compared to a placebo or control group. A hazard function for the treatment group and control group is estimated to determine the total effectiveness of the intervention and the placebo. The difference (positive or negative) between the hazard functions is the benefit or cost of the treatment. Mathematically, effectiveness is measured by the area under the distribution curve or by taking the integral of the hazard function.

2.4.1 Survival Analyses Limits: survival models often are used in clinical decision making, under uncertain conditions, to estimate life expectancies. Kuntz and Weinstein outlined two types of life expectancy biases that may arise in clinical decision modeling: misestimating bias and misspecification bias (Clarke 2004). Both biases potentially can limit the accuracy and validity of any conclusions drawn from a predictive model for survival. Misestimating bias arises because the expected value of a function is not equal to the function itself. For linear functions, the expected value of the function equals the function; however, for nonlinear functions, such as life expectancies and hazard functions, this is not true. 
For most clinical situations, nonlinear functions do not adequately capture the relationship between survival time and life expectancies introducing miss-estimation bias into the model (Kuntz, 1995). Misspecification bias occurs when specifying the function to represent survival outcomes based on the patient's disease and treatment history. There are a number of parametric survival models available to the decision maker, including the Weibull, Makeham-Gomperz, and exponential models. Life expectancies are calculated from the area underneath the survival distribution. When survival distribution does not reflect the patient's conditional survival probability accurately, the life expectancy estimates derived from the distribution will be inherently biased (Collett, 1999, and Kuntz, 1995). 


\subsection{MULTI-COMPARISON and RANKING-SELECTION PROCEDURES}

Recent research has shown that multi-comparison procedures can be combined with ranking and selection procedures $(R \& S)$ for a variety of problems including the manufacturing, medical, and polling examples outlined above (see Nelson 2001). Ranking and selection procedures are statistical methods designed to find the "best" system from a collection of competing alternatives (Nelson 1998 and 2003).

\subsubsection{Different Classes of Screening, Ranking-Selection Procedures:}

R\&S procedures have been expanded over the last 22 years. Kim and Nelson, (2003), pages 110 and 111, stated four different classes of comparison problems, utilizing $R \& S$, which arise in simulation studies. However; we consider that there are six different classes of comparison problems, mainly they are:

1. Selecting the best system(s), of $k$ systems, which has the largest or smallest expected performance measure; there are many procedures have been developed (see Bechhofer 1995, Kim-Nelson 2001, and (Nelson-Miler 1995). For example, Nelson and Miler have developed a Multiple comparison procedure (MCP) combined with the R\&S procedure of Rinott (1978). Rinott procedure is one of the frequently used procedures in $\mathrm{R} \& \mathrm{~S}$. Rinott+MCP utilize a common random number to decrease the variance and to reduce the number of observations required to make a correct selection. (Besides selecting the best system among $\mathrm{K}$, $\mathrm{k} \geq 2$, select a subset of size $m$ containing the best of $\mathrm{K}, \&$ select the $m$ best of K).

2. Comparing all alternatives against a standard (comparison with a standard). The goal of comparison with a standard is to find systems whose expected performance measures are larger (smaller) than a standard and, if there are any, to 
find the one with the largest (smallest) expected performance. For this type of problem, each alternative needs to be compared to the standard as well as other alternative systems. (see Nelson 1998, Nelson 2001, and Kim (2003).

3. Selecting the system with the largest probability of actually being the best performer (multinomial selection). When we are interested in the actual value of performance measure and not the expected value of performance, then the goal is to select the design associated with the largest probability. (see Nelson 1998, and Bechhofer 1995).

4. Selecting the system with the largest probability of success (Bernoulli selection). In Bernoulli selection problems, the basic output from each system on each replication is either one ("success") or zero ("fail") and the best is defined as the system with the largest probability of success. See chapter 7 of Bechhofer et al, (1995), for a inclusive reference.

5. A fifth approach is, by Morrice et al, 1998, to convert multiple performance measures to a scalar performance measure using multiple attribute utility (MAU) theory. MAU theory can be used instead of a costing approach when good cost data are not available. Alternatively, MAU theory can be used to beautify costing information that is considered to be incomplete/ indefinable. This approach combines multiple attribute utility theory with statistical R\&S using the indifference zone approach. And its goal is to select the best project configuration from a set of $\mathrm{K}$ configurations when project performance is measured over multiple performance measures. (Morrice 1998) 
6. There is another formulation; the Bayesian approach: Instead of providing a probability of correct selection, (PCS), guarantee, Bayesian approaches attempt to allocate a finite data resources to maximize the posterior PCS of the selected system. (Chen 2000 and Chick 2001) are two recent references.

A good procedure is one that delivers the desired the probability of correct selection (PCS) efficiently (with minimal simulated data) and is robust to its underlying assumptions. The "best" mainly means the maximum/minimum expected-value (or probability) of performance, such as expected cost. R\&S origins to two papers: "Bechhofer (1954) established the indifference-zone formulation, (IZ), while Gupta $(1956,65)$ is credited with the subset selection formulation of the problem, and both were developed for the inference provided by hypothesis tests for the homogeneity of the $k$ population parameters, usually means.” (Kim 2003)

2.5.2 Multiple Comparisons: in statistics, there are different approaches of comparison such pair-wise comparisons. Case of comparing two alternative systems and case of comparison with a standard are two examples. Moreover, there is what called multiple comparisons, and multiple tests; a researcher would better to make a distinction between them.

Our work, from this point of view, combines multiple comparisons, and rankingselection procedures. Multiple-comparison procedures (MCPs) treat the comparison problem as an inference problem on the performance parameters of interest. MCPs account for the error that arises when making simultaneous inferences about differences in performance among the systems. Usually, MCPs report to the user simultaneous confidence intervals for the differences. 
Multiple Comparisons Procedure approaches the comparison problem by providing simultaneous confidence intervals on selected differences among the systems' parameters. Hsu, 1996, is good reference. MCP forms simultaneous confidence intervals for $\left(\mu_{i}-\max \mu_{s}\right)$, where $\mathrm{s} \neq \mathrm{i}$ and $i=1,2 \ldots . . k$, the difference between each system and the best of the rest. There are other procedures that combine more than approach in one. For example, Nelson (2001), developed what is called NSGS procedure combines a Guptalike subset-selection procedure and a Bechhofer-like ranking procedure. The NSGS is appropriate for terminating simulations or for steady-state simulations when multiple replications are employed.

2.5.3 Multiple Comparisons and Multiple Tests: To make more than one decision in a statistically valid way, multiple inferences are involved. There are numerous alternative solutions for multiple inference problems---“some are very good, some perform reasonably well, and some are of questionable value. The wide variety of methods that are available can make the choice of technique difficult" Westfall et al. (1999). There are various methods, along with their pitfalls and advantages. There are various applications in many areas, including business, medicine, sociology, and engineering.

The proper choice of a multiple inference procedure depends upon researcher inference objectives and data structure. In some statistical software there are procedures for such inferences (e.g. in such as SAS: the GLM, MIXED, and MULTTEST); in several types of problems, they are not particularly well accommodated, and so to fill this gap, there was/is a need for a set of macros and programs. Westfall et al. (1999) introduces some of the new methods, SAS-macros, and capabilities for multiple 
comparisons, allow researchers to carry out multiple inferences in most applications of practical interest.

"Multiple comparisons" usually refers to the comparison of mean values from an experiment with multiple groups. For displays, labeled A, B, and C, using the data to compare display A with display B, A with $\mathrm{C}$, and B with $\mathrm{C}$. This is the classic "multiple comparisons" application; and there is a variety of methods for such analyses (e.g., Tukey's method for comparing means in the SAS PROC GLM for example).

"Multiple testing" on the other hand, concerns a broader class of applications. For example, a clinical trial designed to assess "efficacy" of a pharmaceutical compound might be considered "efficacious" if it reduces fever, or if it speeds recovery time, or if it reduces some pain. Here, there are three tests-a comparison of active compound with placebo for each of the three outcomes. This is an example of "multiple testing."

The distinction between multiple comparisons and multiple tests is that, with multiple comparisons, the researcher typically compares mean values of the same measurement, while with multiple testing, the researcher considers multiple measurements.

Formalism: Technically, the problem of multiple comparisons-multiple testing problem can be described as the potential increase in Type I error that occurs when statistical tests are used repeatedly: If $n$ independent comparisons are performed, the experiment-wide significance level $\alpha$ (alpha) is given by

$$
1-\left(1-\alpha_{\text {per comparison }}\right)^{n},
$$

and it increases as the number of comparisons increases. 
In order to retain the same overall rate of false positives (rather than a higher rate) in a test involving more than one comparison, the standards for each comparison must be more stringent. Intuitively, reducing the size of the allowable error (alpha) for each comparison by the number of comparisons will result in an overall alpha which does not exceed the desired limit, and this can be mathematically proved to be true. For instance, to obtain the usual alpha of 0.05 with ten comparisons requires an alpha of 0.005 for each comparison to result in an overall alpha which does not exceed 0.05 .

There has been a great deal of attention paid to developing better techniques for multiple comparisons, such that the overall rate of false positives can be maintained without inflating the rate of false negatives unnecessarily. Such methods can be divided into general categories (Benjamini 1995):

- Methods where total alpha can be proved to never exceed 0.05 (or other chosen value) under any conditions. These methods provide "strong" control against Type I error, in all conditions including partially correct null hypothesis.

- Methods where total alpha can be proved not to exceed 0.05 except under certain defined conditions.

- Methods which rely on an omnibus or compilation test before proceeding to multiple comparisons. Typically these methods require a significant ANOVA/Tukey range test before proceeding to multiple comparisons. These methods have "weak" control of Type I error

- Empirical methods, which control the proportion of Type I errors, utilizing correlation and distribution characteristics of the observed data. 
The advent of computerized resampling methods, such as Bootstrapping and Monte Carlo simulation, has given rise to many techniques in the latter category. In some cases where exhaustive permutation resampling is performed, these tests provide exact, strong control of Type I error rates; in other cases, such as bootstrap sampling, they provide only approximate control. 


\subsection{MULTI ATTRIBUTES UTILITY THEORY (MAU)}

MAU theory is one of the main analytical tools in the field of decision analysis. Simply, decision analysis is an approach to the solution of problems that are complex to solve informally. A MAU analysis of alternatives (in our research, treatment patterns) helps to identify the alternative that performs well on a majority of performance measures. The MAU methodology for the evaluation of a set of alternatives typically starts with (a) identifying of alternatives and measures, (b) estimating of the performance of the alternatives with respect to the measures, (c) developing utilities-weights for the measures, and (d) evaluating of the alternatives.

The alternatives and the performance measures form a matrix in which each row corresponds to an alternative and each column represents a measure. The cells of the matrix contain estimates of the performance of each alternative on each of the measures. Next step generates a single attribute utility function over each measure that is scaled from 0 to 1 , a weight for each measure, and a multiple attribute utility function derived from the single attribute utility functions and the weights. There is more than one method for assigning weights to the performance measures (Schoemaker 1982). A single attribute utility function is a scoring function that maps a performance measure from its range of possible values to $[0,1]$.

Regular forms of this function include concave, convex, linear, and " $\mathrm{S}$ " shaped for a mixture of the convex and concave functions. One known form for single attribute utility function is

$U(x)=G-H e^{(Y / T)} \ldots .(1)$ 
The quantities $\mathrm{G}, \mathrm{H}$, and $\mathrm{T}$ are parameters that set by the decision maker (in medical literature, it is common to set $\mathrm{G}=\mathrm{H}=1$ for patients, as patients are risk avoider.

In the analysis utility theory involves gathering the measures into a "UnifiedMeasure" of the desirability of each alternative. Utility theory provides the basis for the appropriate approach to combined the apparently unlike measures.

If the decision maker's preferences are consistent with some special independence conditions, then a multiple attribute utility model $u\left(x_{1}, x 2, \ldots, x_{n}\right)$, where $x i$ represents the level of performance on measure $i$, can be decomposed into an additive, multiplicative, or other well structured forms that simplify assessment. An additive multiple attribute utility model can be represented as follows:

$$
\left.u\left(x_{1}, x_{2} \ldots \ldots, x_{n}\right)=\sum_{i=1}^{n} w_{i} u_{i}\left(x_{i}\right) \quad 12\right)
$$

Where $u_{\mathrm{i}}($.$) is a single attribute utility function over measure \mathrm{i}$ that is scaled from 0 to $1, w_{i}$ is the weight for measure $\mathrm{i}$ and sum of $w_{i}=1, \mathrm{I}=1,2, \ldots \mathrm{n}$.

If the decision maker's preference structure is not consistent with the additive model (2), then the following multiplicative model may be used, which is based on a weaker independence condition:

$$
1+k u\left(x_{1}, x_{2}, \ldots, x_{n}\right)=\prod_{i=1}^{n}\left[1+k k_{1} u_{i}\left(x_{1}\right)\right]
$$

Where $u_{\mathrm{i}}($.$) is also a single attribute utility function scaled from 0$ to 1 , the $k_{i}$ 's are positive scaling constants satisfying $0 \leq k_{i} \leq 1$, and $k$ is an additional scaling constant that characterizes the interaction effect of different measures on preference. Methods for determining the value $k$ can be found in Keeney and Raiffa, 1976. As a special case when 
sum of $k_{i}=1$, the multiplicative model (3) reduces to the additive model (2) (Keeney and Raiffa, 1976).

\subsection{SIMULATION MODELS}

David Kelton et al, (Kelton 2001), defined simulation as: "a broad collection of methods and applications to mimic the behavior of real systems, usually on a computer with suitable software". Jerry Banks et al, (Banks 1995), had defined simulation as " the imitation of the operation of a real-world process or system over time".

\subsubsection{Simulation models}

- Monte Carlo Simulation: Monte Carlo models are prearranged likewise to Markov models, but their outcome evaluations are determined by repeated iterations of the model based on random assignment of model parameter. Monte Carlo simulation (traditional simulation) techniques use repeated random sampling from the probability distributions assigned to each of the crucial primary variables the patients flow of a treatment to arrive at output probability distribution for a given medication strategy. Monte Carlo simulation is a forward-looking technique based on a predetermined operating strategy; as such, it may be an appropriate model for path-dependent or history-dependent problems. (Jaafari, 2003)

- Process Simulation: "process simulation permits investigation of an intervention concept to generate the expected units of output. It also permits investigation of the interrelationships among constituent parts of a medication study. Process simulation can be a prior to pilot medical-project operation." (Jaafari, 2003) 
Process Simulation should run before startup or charge of the actual arrangement or, with the use of a spare offline system, anytime.

- There is what is called Nevada Simulation; NEVADA Simulation, developed by David J. Bryg, (1992); it is very competitive compared to Monte Carlo simulation in terms of accuracy and speed. NEVADA Simulation employs numerical integration to calculate functions of random variables. This technique contrasts to Monte Carlo simulation, which uses sampling to calculate functions of random variables.

In our work, we utilize Monte Carlo simulation techniques.

Simulation has advantages, (Jerry Banks, et al 1996): First, simulation can be a method of last alternative for problems that are mathematically intractable by any other techniques. Second, even for problems that are mathematically tractable, simulation can often provide a higher level of detail than can other techniques. Third, simulation can sometimes provide (approximate) answers at a smaller cost (or effort) to some problems which are fully tractable mathematically but whose solution may be cumbersome and time consuming.

Other advantages of simulation include that it permits modification or design of medical intervention systems by trial and error, allows for easy exploration of the system's sensitivity to changes in the input parameters, and provides a highly controllable environment for experiments. Simulation can be valuable to the operations researcher as a means of testing the applicability and validity of mathematical models and expressions.

2.7.1 Simulation Limits, the disadvantages of simulation as a technique are also primarily due to the fact that simulation is basically an experimental approach to solving 
problems (Jerry Banks, et al 1996). As a result it is difficult to develop cause-and-effect relationships through simulation, especially when the system under consideration requires the specification of many input parameters and involves complex interactions. Finally, as with all experimental results (and, actually, more so) the statistical analysis of simulation results is difficult. What is the effect of the starting conditions of the simulation on the final results? What is the statistical confidence that can be attached to the results? 


\subsubsection{Output analysis for Terminating Simulations for the Fixed-Sample-size}

Consider output performance measure X, e.g., the average QALY for a patient in the single-treatment system. Let $\mathrm{Xj}$ be the value of the performance measure in the jth independent simulation replication, $\mathrm{j}=1,2, \ldots, \mathrm{n} . \mathrm{Xj}$ are IID observations of the performance measure

Estimating the mean:

The fixed-sample-size procedure for $\mathrm{m}=\mathrm{E}(\mathrm{X})$

$$
\begin{aligned}
& \text { Choose } \mathrm{n} \text { and make Independen } \mathrm{t} \text { Replicatio } \mathrm{ns} \text { of the simulation } \\
& 1-\alpha \text { confidence interval for } \mu \text { is } \\
& \bar{X}(n) \pm t_{n-1,1-\alpha / 2} \sqrt{\frac{S^{2}(n)}{n}} \\
& \text { where } \bar{X}(n)=\frac{1}{n} \sum_{i=1}^{n} X_{i} \\
& \text { and } S^{2}(n)=\frac{\sum_{i=1}^{n}\left(X_{i}-\bar{X}(n)\right)^{2}}{n-1}
\end{aligned}
$$

Coverage: $t$-interval assumes normality of X1, X2, .. Xn. Robustness depends on the sample size and on the simulation model. Effect of departures from normality may be assessed with comparison to known results.

Obtaining specified error: Absolute error $\beta=|\bar{X}(n)-\mu|$

We make sufficiently many runs so that the half-length of the $1-\alpha$ confidence interval is equal to or less than $\beta$ 


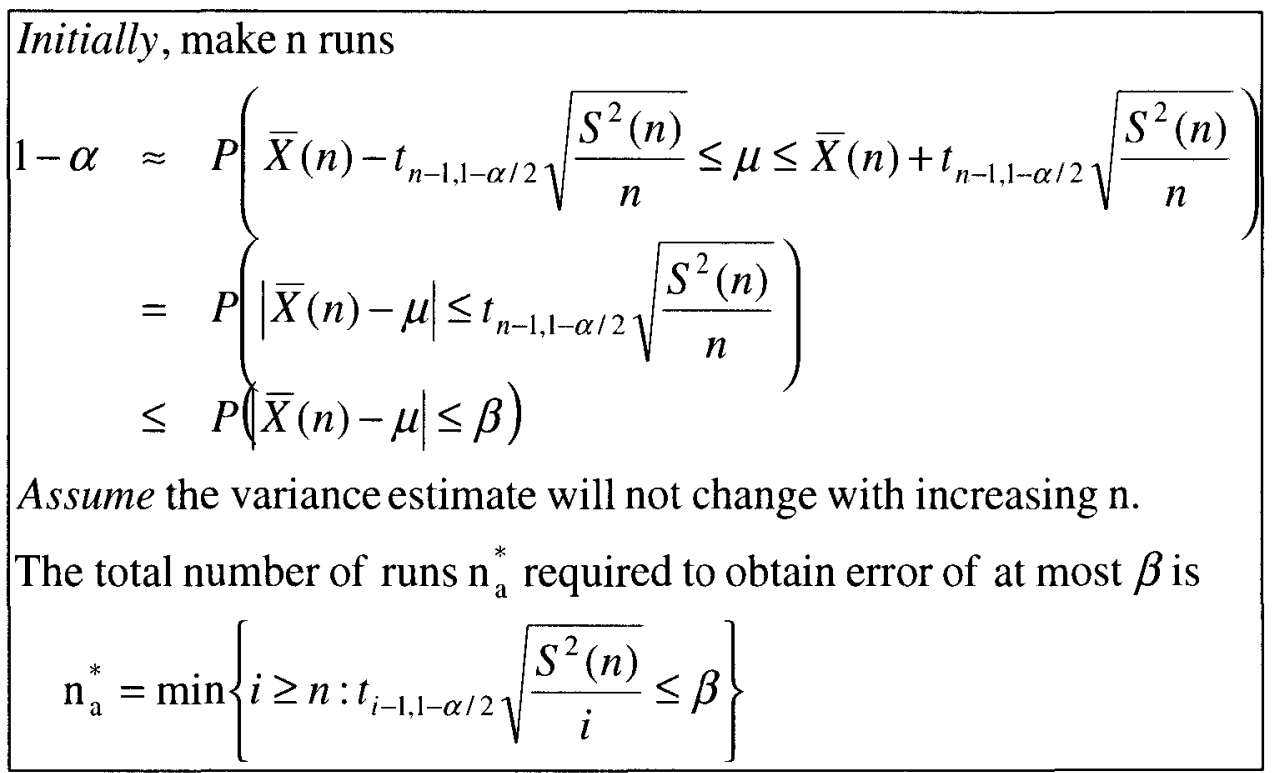

\section{Obtaining specified error: Relative error}

$$
\gamma=|\bar{X}(n)-\mu| /|\mu|
$$

We make sufficiently many runs so that the half-length of the 1- $\alpha$ confidence interval, divided by the estimate of the mean, is equal to or less than $\gamma$

A sequential procedure

Choose an initial number of replications $\mathrm{n}_{0} \geq 2$ and define the confidence interval half - length as

$$
\delta(\mathrm{n}, \mathrm{a})=t_{n-1,1-\alpha / 2} \sqrt{\frac{S^{2}(n)}{n}}
$$

0. Make $\mathrm{n}_{0}$ replications and set $\mathrm{n}=\mathrm{n}_{0}$

1. Compute $\overline{\mathrm{X}}(\mathrm{n})$ and $\delta(\mathrm{n}, \mathrm{a})$ using $\mathrm{X}_{1}, \mathrm{X}_{2}, \ldots, \mathrm{X}_{\mathrm{n}}$

2. If $\delta(\mathrm{n}, \mathrm{a}) /|\overline{\mathrm{X}}(\mathrm{n})| \leq \gamma$, use $\overline{\mathrm{X}}(\mathrm{n})$ as estimate of $\mu$ and stop.

The $1-\alpha$ confidence interval for $\mu$ is $[\overline{\mathrm{X}}(\mathrm{n})-\delta(\mathrm{n}, \mathrm{a}), \overline{\mathrm{X}}(\mathrm{n})+\delta(\mathrm{n}, \mathrm{a})]$

Otherwise set $\mathrm{n}=\mathrm{n}+1$ and go to step 1 .

Recommendations:

(1) $n_{0}$ is recommended to be larger than or equal to 10

(2) $\gamma n_{0}$ is recommended to be less than or equal to 0.15 


\subsubsection{Output Analysis for Terminating Simulations When $n$ can be determined}

When $n$ can be determined many methods may be applied to select best system, such as multiple comparison, ranking and selection, interactive analysis, etc.

Example (1) (Goldsman, Nelson 1998): suppose that Airline-Reservation System, Consider $k=4$ different systems; and the objective is to maximize E[Time To Failure]. From experience, E[TTF] roughly 100,000 minutes for all four systems; and the indifference zone is 3000 minutes.

- Solution by Interactive Analysis: an estimation approach: It considers 4 point estimators for E[TTF]'s; and estimates their standard errors; the goal is a vague, but well-founded, sense of confidence in the selected system; IA here contains the explicit confidence-interval judgment.

The Method: $m=$ number of micro-replication, $b=$ number of macroreplication; $n=b m$; the point estimator for $\mu i, i=1,2,3,4$ (the $\mathrm{E}[\mathrm{TTF}]$ of system $i)$ :

$$
\bar{Y}_{i}=\frac{1}{b} \sum_{j=1}^{b} \bar{Y}_{i j}=\frac{1}{n} \sum_{j=1}^{b} \sum_{l=1}^{m} Y_{i j l}
$$

With associated sample variance of the macro-replication estimators :

$$
S_{i}^{2}=\sum_{j=1}^{b} \frac{\left(\bar{Y}_{i j}-\bar{Y}_{i}\right)^{2}}{(b-1)} \quad s e_{i}=\frac{S_{i}}{\sqrt{b}}
$$

The Assumptions: People choose $\alpha$ arbitrarily and small b \& $\alpha$ can lead to large $t$ values; Choose $10<b<30$ is often wise (Goldsman, Nelson, 1998); If the value of $\mathrm{b}$ is reasonably large, the effects of sequential sampling are negligible. 
Initial run is designed just to gain a sense of the magnitude of the required production experiment in terms of time per replication and number of replications System: standard error (se) $=21000$, and $b=m=5$; indifference value $=3000$; so the (se) will need to drop to at most 1500 ; this means that the "worst-case" is 5000 replications.

- Solution by Ranking \& Selection: it is to select the best system from a set of competing systems. The probability of a correct selection will be at least some user-specified value. For example, when variances are unknown two-stage $R \& S$ procedure such as Dudewicz-Dalal, (1997), and Rinott's (1978) procedure required; the two stages of sampling guaranteed PCS. The normal means procedure of Rinott, (1978):

The Method: Ordering $u_{[1]} \leq u_{[2]} \leq \ldots \leq u_{[k]}$; the two best $u_{[k]}, u_{[k-1]}$ If $u_{[k]}-u_{[k-1]}$ is very small, less than $\delta>0$; it wouldn't matter which one we chose as best $(\delta=3000)$. We take $\mathrm{P}^{*}=0.9$ in our example; $\mathrm{P}(\mathrm{CS}) \geq \mathrm{P}^{*}=0.9$

The first-stage sample means and sample variances

$$
\bar{Y}_{i}^{(1)}=\sum_{j=1}^{b_{0}} \frac{\bar{Y}_{i j}}{b_{0}} \quad S_{i}^{2}=\sum_{j=1}^{b_{0}} \frac{\left(\bar{Y}_{i j}-\bar{Y}_{i}^{(1)}\right)^{2}}{\left(b_{0}-1\right)}
$$

The sample variances are used to determine the number of macro-replications which must be taken in the second stage

$$
b_{i}=\max \left\{b_{0},\left\lceil\left(\frac{h S_{i}}{\delta}\right)^{2}\right\rceil\right\}
$$

(a) $h$ is a function in the number of replications $n$, the number of systems $k$, and $\delta$. To find $h$ value, named $g$, see Bechhofer, (1995), page 62 . 
(b) So $b_{i}-b_{0}$ additional observations must be taken. Finally we get grand means $\bar{Y}_{i}=\sum_{j=1}^{b_{i}} \frac{\bar{Y}_{i j}}{b_{i}}, i=1,2, \ldots, k$ and select the system with largest $\bar{Y}_{i}$

(c) The macro-replication estimators, $\overline{Y_{i 1}}, \overline{Y_{i 2}}, \ldots, \overline{Y_{i b_{i}}}$ from the $\mathrm{i}^{\text {th }}$ system are assumed to be i.i.d. with expectation. If the number of micro-replications $m$ is large enough the Central Limit Theorem (CLT) yields approximate normality for the macro-replication estimators. For sampling $b_{0}=20$, intend to be used as the first stage:

Table 1: Pilot experiment on Ranking \& Selection

\begin{tabular}{|c|c|c|c|c|}
\hline $\mathrm{i}$ & 1 & 2 & 3 & 4 \\
\hline $\mathrm{Y}^{(1)}$ & 108286 & 107686 & 96167.7 & 89747.9 \\
\hline$S_{i}$ & 29157.3 & 24289.9 & 25319.5 & 20810.8 \\
\hline$s_{i}$ & 6519.8 & 5431.4 & 5661.6 & 4653.4 \\
\hline$b_{i}$ & 699 & 485 & 527 & 356 \\
\hline
\end{tabular}

Table 2: Pilot experiment on Ranking \& Selection Result

\begin{tabular}{|c|c|c|c|c|}
\hline $\mathrm{i}$ & 1 & 2 & 3 & 4 \\
\hline$Y_{i}^{(1)}$ & 110816.5 & 106411.8 & 99093.1 & 86568.9 \\
\hline$s e_{i}$ & 872 & 1046.5 & 894.2 & 985.8 \\
\hline
\end{tabular}

Final Results: For the case $\mathrm{k}=4, \mathrm{P}^{*}=0.9$; and $\mathrm{h}=2.720$ (Bechhofer, (1995), page 62); for system 2 , we needed to take 465 additional macro-replications in stage 2 . We are at least $90 \%$ sure that we have made the correct selection $\left(\mathrm{u}_{(1)}-\mathrm{u}_{(2)}>\delta=3000\right)$ 


\section{Output analysis for terminating simulations:}

Solution by Multiple comparisons: There is no distinction between micro and macro-replication $Y_{i j}=u_{i}+\varepsilon_{i j} \quad \varepsilon_{i j}$ are iid normal $\left(0, \sigma^{2}\right)$

$$
\begin{aligned}
& \left(\bar{Y}_{i}-\max _{l \neq i} \bar{Y}_{l}-d s \sqrt{\frac{2}{n}}\right)^{-},\left(\bar{Y}_{i}-\max _{l \neq i} \bar{Y}_{l}+d s \sqrt{\frac{2}{n}}\right)^{+} \\
& s^{2} \text { is pooledestimatorof } \sigma^{2} \quad d=d_{1-\alpha, k(n-1), k} \\
& x^{-}=\min \{0, x\}, x^{+}=\max \{0, x\}
\end{aligned}
$$

Lower endpoint is 0 , then system $i$ is the best system Upper endpoint is 0 , then system $i$ is bad system

Assumption: Data from one-way analysis of variance; use different random number seed to generate sample, so the relationships between samples are independent. Sample from normal and common variance

$$
Y_{i j} \sim \text { normal distributi on } \operatorname{VAR}\left(Y_{i j}\right)=\sigma^{2} \forall i, j
$$

\section{Pilot experiment (Batch mean)}

Because $n$ replications need to be large that samples may be normally distributed, so use batch mean method to generate sample.

Using batch mean will tend to form the normal distribution, but the drawback is the lose of degree of freedom

$$
\begin{aligned}
& Y_{i 1}, Y_{i 2}, \ldots, Y_{i n} \text { become to batch mean formal } b_{i} m_{i}=n \\
& \bar{Y}_{i l}=\frac{1}{m_{i}} \sum_{j=1}^{m_{i}} Y_{i,(l-1) m_{i}+j}
\end{aligned}
$$


For $n=200, b=40$ and $m=5$

\section{Table 3}

\section{A Pilot Experiment on MCP}

\begin{tabular}{|c|c|c|c|}
\hline $\mathrm{i}$ & Lower limit & $\bar{Y}_{i}-\max _{l \neq i} \bar{Y}$ & Upper limit \\
\hline 1 & -11349 & 7091 & 25530 \\
\hline 2 & -31242 & -12802 & 5638 \\
\hline 3 & -25530 & -7091. & 11349 \\
\hline 4 & -36172 & -17732 & 708 \\
\hline
\end{tabular}

Since all system contained 0 no system could be declared to be the best. $S=92449$, $\mathrm{d}=2.078, \mathrm{n}=8100$ for final result.

$$
n \geq\left(\frac{\sqrt{2} d_{1-\alpha, k(n-1), k} \sigma}{\delta}\right)^{2} \quad \text { Let half interval of } \mathrm{CI} \leq \delta
$$

Using Bartlett's test for equality variance, the result is different, so set $b_{1}=b_{2}=b_{3}=100$, and $b_{4}=150$, let variance is same.

\section{Table 4}

Final Result on a Pilot Experiment on MCP

\begin{tabular}{|c|c|c|c|}
\hline $\mathrm{i}$ & Lower limit & $\bar{Y}_{i}-\max _{l \neq i} \bar{Y}$ & Upper limit \\
\hline 1 & 0 & 5288 & 8284 \\
\hline 2 & -8284 & -5822 & 0 \\
\hline 3 & -12747 & -9751 & 0 \\
\hline 4 & -25409 & -22675 & 0 \\
\hline
\end{tabular}

System 1 is the best. 
Table 5: Comparison Methods' Differences and Similarities

\begin{tabular}{|c|c|c|c|c|c|}
\hline Method & $\begin{array}{c}\text { Micro } \\
\text { replication }\end{array}$ & $\begin{array}{c}\text { Marco } \\
\text { replication }\end{array}$ & CI & Stage & Assumption \\
\hline IA & Yes & Yes & No & One & $\begin{array}{c}\text { Sample from } \\
\text { iid normal }\end{array}$ \\
\hline R\&S & Yes & Yes & No & Two & $\begin{array}{c}\text { Sample from } \\
\text { iid normal }\end{array}$ \\
\hline MCP & No distinguish & Yes & Two & Common variance \\
& & & & but will check it. \\
\hline
\end{tabular}

It is difficult to have a common variance in $n$ systems/models, so $\mathrm{R} \& \mathrm{~S}$ have an advantage on MCP. R\&S can handle comparison with not common variance.

Table 6: $\underline{\text { Comparison Methods Advantages and Disadvantages }}$

\begin{tabular}{|r|l|l|}
\hline Method & \multicolumn{1}{|c|}{ Advantage } & \multicolumn{1}{|c|}{ Disadvantage } \\
\hline TA & Noncompetitive system are eliminated quickly & Lack of precise confidence \\
\hline R\&S & Guarantee $\mathrm{P}\{\mathrm{CS}\} \geq \mathrm{P}^{*}$ & When better system is not one, \\
& & Take more observations is \\
\hline MeP & It provides inference about not only the best & It need rather/somewhat \\
& system, but also relationships among all the & strict/severe distributional \\
systems. & assumption \\
\hline
\end{tabular}


We will use Rinott's (1978), it used when variances are unknown; it is two-stage R\&S procedure; the two stages of sampling guaranteed PCS. Rinott's (1978) procedure: Satage I: take a random sample of no observation from each system i.

Stage II: calculate $\mu$ and variance $S^{2}$ for each system; take additional observation from each system I where,

$$
N_{i} \quad \max \left\{n_{0},\left\lceil\left(\frac{h S_{i}}{\delta}\right)^{2}\right\rceil\right\}
$$

Where $h=h\left(k, l-\alpha, n_{0}\right)$ is a constant determined by $\mathrm{k}$, the number of systems being compared; $1-\alpha$, the desired confidence level; and $n_{0}$, the number of first stage observations used to produce the variance estimator, $(\mathrm{Si})^{2}$. The constant $h$ increases in $k$, and decreases in $\alpha$ and $n_{0}$. The experiment design factor that is under our control is $n_{0}$, and it is recommended to be at least 20 . Calculate new $\mu$ and variance $S^{2}$ for each system based on the combination results of stage I, and II. Select the treatment that has max $\boldsymbol{\mu}$ to be the best system.

Our simulation model is not a steady-state simulation model; it is a terminating simulation. We assume that performance measures have no correlations, i.e. we suppose that the performance measures are utility independent; however, our model can handle performance measures which are utility dependent or utility independent.

Nelson, et al. (2001), developed a procedure related to Rinott called NSGS procedure, it is appropriate when there are large number of replications, and for terminating simulations or for steady-state simulations when multiple replications are employed. The NSGS procedure requires that the output data from each system are i.i.d. normal, and that outputs across systems are independent, which leaves out CRN. Nelson 
et al. (2001) showed that the NGSG procedure can handle a relatively large number of systems because the first-stage screening is pretty tight. Nelson et al. (2001) provide a revised version of the NGSG procedure, the Group-Screening procedure, in which one can avoid simulating all the systems simultaneously. But, in medical intervention, there is not large number of systems to be tested for specific recurrent disease. 


\section{CHAPTER III}

\section{METHODS}

\section{An Enhancement of the Stochastic Tree Model for Medical Decision}

\section{Making (Model I)}

\section{Model I. Background}

While conventional decision models are an attractive way in which to model problems under uncertainty, they cannot investigate disorders that are recurring in nature. Stochastic tree models were developed to deal with cyclic disorders. Some stochastic tree models were developed to deal with recurrent diseases and to utilize a specific probability distribution for health states sojourn (see Hazen 1992-2001). However, some disorders are best represented by probability distributions other than that allowed by stochastic trees. Furthermore, such stochastic tree models cannot correct and consider boundary differences of the experimental treatment circumstances and conditions and the standard treatment circumstances and conditions. In this chapter we utilize the Weibull Accelerated Failure Time model (WAFT) to extend the stochastic tree modeling technique. This new technique is a novel way in which to analyze cyclic disorders and recurrent diseases. 


\section{Model I. Introduction}

Decision trees represent a functional way in which to analyze problems under uncertainty and provide a mechanism to analyze decisions under risk (Detsky, 1996-97). Conventional decision trees examine complex issues composed of discrete sequential events; such as, undergoing tests, receiving the tests results, and deciding on the optimal medical management. Conventional decision trees do not completely represent "the real world" since they cannot investigate disorders that are cyclic in nature (Jaafari, 2003). As a result, researchers have developed methodologies, via Markov processes, allowing for the examination of cyclic disorders. For example, Hazen developed a stochastic tree model, combining features of continuous-time Markov chains with those of conventional decision trees in order to analyze cyclic disorders (Hazen, 1992-2001).

Hazen's stochastic tree model allows health state transitions to occur at any instant. In Hazen's stochastic tree technique, the time spent in any health state is restricted to a low-dimensional preference summary (e.g. memory less, Markov, and semi-Markov) for ease utility recursive computations. The notation of preference summary: memory less is an updatable preference summary in which conditional preference depends not at all on the past; the Markov summary is conditional preference depends only on the most recent state; the semi-Markov summary is conditional preference depends on the most recent state and its stay time. Hazen's stochastic tree modeling technique can only utilize an exponential distribution for health state sojourn. Noticeably, some re current diseases can be best represented by distributions such as Weibull distribution, Gamma distribution, Log-Logistic distribution, Log-Normal distribution, Coxian, and Quadratic distribution (Collett, 1999). In addition, the stochastic 
tree modeling technique does not have a method to correct differences between experimental conditions and the corresponding conditions of a current treatment.

Quality-adjusted life years (QALYs) are the measure of health benefit to an individual that reflects the gain in expected utility for the individual. QALYs can be used to represent utility only if individuals are willing to trade off years of life in a given health state for fewer years at an ideal health state at a constant rate, irrespective of the number of years spent in the state. In addition, it would be beneficial if these methodologies integrate an individual's specific health conditions (e.g. smoking history, body mass index).

In this part of our study, we extend Hazen's stochastic modeling technique, which incorporates the memory-less exponential distribution by utilizing the Weibull Accelerated Failure Time model (WAFT). The WAFT model is memory-less when Weibull's shape parameter equals to one, but also integrates specific health conditions at the individual level. This new technique may not only provide a novel way in which to study QALYs, it may also provide a novel way in which to analyze recurrent diseases. 


\section{Model I. Specifications and Design}

Model I is an analytical model: An Enhancement of Hazen's Stochastic Tree Model Using Weibull Accelerated Failure Time Model for Medical Decision Making in Recurrent Diseases/Injuries Specification

Use of Survival Analysis: Systems that "decay" over time can be modeled using a survival or hazard function. As a result survival analysis is a useful method in which to model treatment effectiveness and cost. In clinical trials, treatment efficacy often is compared between a placebo and control group. A hazard function for the treatment group and the control group can be estimated to determine the total effectiveness of both the intervention and the placebo. The difference between the respective hazard functions represents the benefit or loss associated with the treatment.

Survivor and Hazard Functions: The survival time of a particular individual, $t$, can be regarded as the value of the random variable $T$ representing survival time. Thus, the different values that $T$ can take correspond to a probability distribution function, $f(t)$. The distribution function of $T$ is then given by:

$$
F(t)=P(T<t)=\int_{0}^{t} f(t) d u
$$

The survivor function $S(t)$ is defined as the probability that the survival time is greater than or equal to $t$, that is,

$$
S(t)=P(T \geq t)=1-F(t)
$$

For recurring sickness or weakness due to illness, the morbidity ratio as a function of that recurrence can be regarded as a survivor function for use in survival analysis. The hazard function is then defined as the probability that a patient's sickness/weakness level 
will reach some defined failure criterion (a morbidity ratio reduction of $\mathrm{m} \%$ at time $t$, conditional on the patient having survived to time t). Thus the hazard function is the instantaneous harm rate. Hence from (Collett, 1999, page 12),

$$
h(t)=\lim _{\delta t \rightarrow 0} \frac{P(t \leq T<t+\delta t \mid T \geq t)}{\delta t}
$$

For two events $\mathrm{A}$ and $\mathrm{B}$ with $\mathrm{P}(B) \neq 0$, the conditional probability of A given B can be expressed as:

$$
P(A \mid B)=\frac{P(A \cap B)}{P(B)}
$$

Therefore,

$$
P(t \leq T<t+\delta t \mid T \geq t)=\frac{P(t \leq T<t+\delta t)}{P(T \geq t)}=\frac{F(t+\delta t)-F(t)}{S(t)}
$$

Hence,

$$
h(t)=\lim _{\delta t \rightarrow 0} \frac{P(t \leq T<t+\delta t \mid T \geq t)}{\delta t}=\lim _{\delta t \rightarrow 0} \frac{F(t+\delta t)-F(t)}{\delta t} \cdot \frac{1}{S(t)}=\frac{F^{\prime}(t)}{S(t)}
$$

The derivative of $F(t)$ with respect to $t$ is $f(t)$. Hence, the hazard function h $(t)$ used in survival analysis is defined as

$$
h(t)=\frac{F^{\prime}(t)}{S(t)}=\frac{f(t)}{S(t)}=-\frac{d}{d t}(\ln S(t))
$$

And,

$$
f(t)=h(t) * S(t)=\left[-\frac{d}{d t}(\ln S(t))\right]^{*} S(t)=-\frac{d}{d t} S(t)
$$


Weibull Model: the Weibull distribution plays a central role in the analysis of survival data. Introduced by Weibull in 1951, in industrial reliability testing, this distribution is fundamental to the parametric analysis of survival data. Research has demonstrated that improving health associated with a medical intervention can be most accurately represented by a survivor function using a Weibull density function (Collett, 1999). The Weibull approach uses a "recursive" approach. That is, harm is updated throughout a patient's life, with the morbidities/weaknesses in period $n$ adjusted for the patient's conditions in period $n$, and accumulated illness harm through period $n-1$. The Weibull model provides a better description of the non-linear accumulation of harm of the sickness levels when compared to traditional linear hypothesis of cumulative harm. The Weibull density function has the following form

$$
f(t)=\lambda t^{\gamma-1} \exp \left(-\lambda t^{\gamma}\right), \quad \text { where } 0 \leq t \leq \infty
$$

$\lambda$ (or $\mathrm{L}$ ) is the scale parameter and $\gamma$ (or $\mathrm{G}$ ) is the shape parameter; with both parameters being non-negative. Figure 1 Three different curves of Weibull density function:

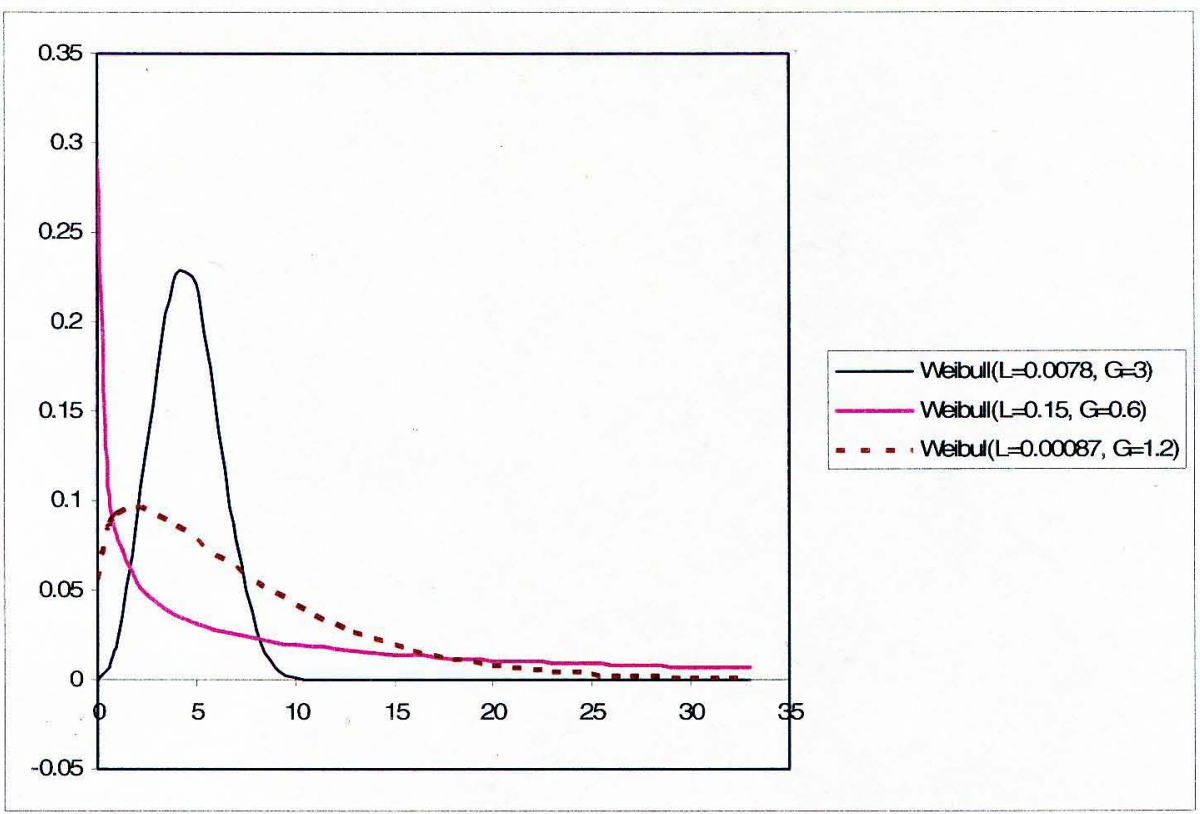


When disease harm progresses, experimental morbidity tests follow a Weibull distribution with a baseline hazard function of the form

$$
h_{\text {Exper }}(t)=\lambda \mu^{\gamma-1}
$$

Accelerated Failure Time Model: the accelerated failure time model is a general model for survival data, in which explanatory variables measured on an individual, are assumed to act multiplicatively on the time-scale. This means that the models can be interpreted in terms of the speed of progression of a disease, an interpretation that has immediate intuitive appeal.

One interpretation of this model is that the lifetime of an individual on a new treatment is a multiple of the lifetime that the same individual would have experienced under a standard treatment by a constant. When the phenomenon of concern is death, values of less than unity correspond to acceleration in the time to death of an individual assigned to the new treatment. The standard treatment would then be the more suitable in terms of promoting longer life. Conversely, values of greater than one occur when the effect of the new treatment prolong life. In these circumstances the new treatment would be viewed as superior to the standard. The parameter is therefore termed the acceleration factor.

The effect, due to different boundary conditions, is to increase or decrease the harm based on each of the patient's conditions. Under this assumption, the probability that a patient in the experimental group survives beyond time $t$ is the probability that a patient in the non-experiment survives beyond time $\Phi t$, where $\Phi$. is an unknown, positive constant. Thus, the $\Phi$ term is analogous to the shift factor, a correction factor applied to 
the patient in the experimental group to obtain the corresponding patient life in the nonexperimental group, and is caused by the differences of boundary conditions between experimental and non-experimental life.

Now letting $S_{\text {Exper }}(t)$ and $S_{\text {Non-Exper }}(t)$ be the survivor functions for some experimental treatment and the current standard of care, respectively. Then, the accelerated failure time model specifies that: $f_{\text {Exper }}(\mathrm{t})=f_{N_{\text {Non_Exper }}}(\Phi \mathrm{t})$ for any value of the survival time t.

Following the relationship between the survivor function $S(t)$, probability density function $f(t)$ and hazard function $h(t)$, the relationship between the density and hazard functions can be derived as follows:

The derivative with respect to time of (2) above,

$$
\frac{d S_{\text {Exper }}(t)}{d t}=\frac{d S_{\text {Non }- \text { Exper }}(\Phi t)}{d t}
$$

Then we have

$f_{\text {Exper }}(\mathrm{t})=\Phi f_{\text {Non_Exper }}(\Phi \mathrm{t})$

According to the definition of the hazard function $\frac{f_{\text {Exper }}(t)}{S_{\text {Exper }}(t)}=\frac{\Phi f_{N_{\text {Non_Exper }}}(\Phi t)}{S_{N_{n} n_{-} \text {Exper }}(\Phi t)}$ We obtain

$h_{\text {Exper }}(\mathrm{t})=\Phi h_{\text {Non_Exper }}(\Phi \mathrm{t})$

The parameter $\Phi$ must be non-negative; therefore, it is convenient to set $\Phi$ as an exponential function.

The Weibull Accelerated Failure Time Model: experiment is extremely important as a part of the medical intervention design. The information obtained from the experiment must be applied to predict the effectiveness of a proposed treatment. 
However, it is inefficient and very difficult to make the boundary conditions of the experimental treatment exactly match those of the standard treatment.

As a consequence, a correction factor, " $\Phi$ ", is needed to correct for the difference in boundary conditions. To predict the patient performance, it is important to understand every feature of the correction factor. It should be noted that the comparison between original data curves and experiment curves needs to be made under the same test conditions. These conditions, if not the same, should be as similar as possible to eliminate potential bias.

Under the assumption, if the health decline process of an experimental treatment is a Weibull function with scale parameter $\lambda$ and shape parameter $\gamma$; the hazard function of the accelerated failure time model for the standard of care can be represented as the following:

$$
h_{\text {Exper }}(t)=\Phi h_{\text {Non_Exper }}(\Phi t)=\Phi \lambda \gamma(\Phi t)^{\gamma-1}=\Phi^{\gamma} \lambda \mathcal{H}^{\gamma-1}
$$

and its survivor function can be defined as

$$
S_{\text {Exper }}(t)=\exp \left(-\int_{0}^{t} h_{\text {Exper }}(u) d u\right)=\exp \left(-\lambda \Phi^{\gamma} t^{\gamma}\right)
$$

which is a Weibull function with scale parameter $\lambda \Phi^{\gamma}$ and shape parameter $\gamma$ Having, $f(\mathrm{t})=$ hazard function $(t) *$ Survival function $(t)$, then:

$$
f(t)=h(t) * S(t)=\left(\Phi^{\gamma} \lambda \mu^{\gamma-1}\right) * \exp \left(-\lambda \Phi^{\gamma} t^{\gamma}\right) \ldots . .(5)
$$

Setting $\Phi \mathrm{i}=\exp \left(\sum_{j} \beta_{j} x_{j i}\right)$, the Accelerated Failure Time Model (AFTM) is a general model for survival data, in which explanatory variables $x_{i}$ measured on an 
individual are assumed to act multiplicatively on the time-scale and therefore affects the rate at which an individual proceeds along the time axis.

This distribution has both the proportional hazards property and accelerated failure time property. The advantage of the Weibull Proportional Hazard model is that it can represent all the various experimental situations with an integrated equation in which the parameters can be easily estimated with linear regression. The AFT model introduces a method to correct for the boundary difference between experimental treatments and the standard of care. The Weibull accelerated failure time model is proposed as a way to bridge the gap caused by the difference of boundary conditions between medical nonexperiment results and the actual outputs when a new medical intervention applied on patient.

Stochastic Tree: for enhancing the medical decision methodology, Beck and Pauker (1983) extended medical decision tree methodology to consider discrete-time Markov process-based models; which is helpful when the timing of a treatment is a critical variable. Recently, Hazen $(1992,93,96,98, \& 2002)$ has developed stochastic trees as a technique for solving continuous-time Markov cycle trees.

Hazen et al, 1992-2002, in the medical decision making field, has developed a an approach that combines features of continuous-time Markov chains with those of decision trees and that enable time to be modeled as a range where health state transitions can occur at any instant. "It can also accommodate patients' preferences regarding risk and quality of life. The stochastic tree concerns it self with the recursive evaluation of utility function, that it is the calculation of an expected utility measure using iterative methods (CYCLIC) similar to the method of successive approximations (value iteration) 
in the stochastic Dynamic Programming”.

Stochastic Tree Limits: the stay in any health state is restricted to lowdimensional preference summary (e.g. Markov preference summary) for the recursive computations and to fit with the Markov process property. So, Hazen's stochastic tree model was created with respect to exponential distribution only; nevertheless, there are other survival data should be represented in different sojourn (survival models) distributions, such as Weibull distribution, Gamma distribution, Log-Logistic distribution, Log-Normal distribution, Coxian, Quadratic, and Bathtub distribution that could be used (Collett, 1999).

Stochastic tree rollback: In 1996 Hazen and Pellissier proved that recursive procedures offer computational advantages for stochastic trees which are large and/or cyclic. They note that in general, the recursion is too computationally challenging to be useful, unless there are convenient preference summaries such as the memoryless property of the exponential distribution (i.e. the computations recursively depend on data one step only in the past, or depend on data of the first level of health states before the current state). Stochastic tree rollback is particularly simple with quality-adjusted duration as the outcome measure (see Hazen-Pellissier, Hazen-Pellissier-Chang 1996). To represent this, Hazen considered a schematic representation of a stochastic fork:
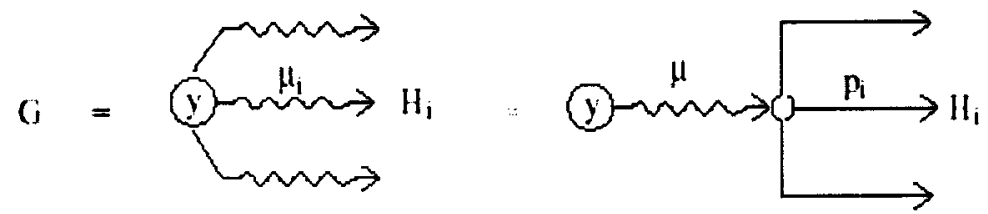

The stochastic fork, above, was formed by competing rates $\mu_{\mathrm{i}}>0$ at state $\mathrm{y}$, where $\mu=\sum_{\mathrm{i}} \mu_{\mathrm{i}}$ and $\mathrm{p}_{\mathrm{i}}=\mu_{\mathrm{i}} / \mu \mathrm{H}_{\mathrm{i}}$ is itself a stochastic subtree for which the utility $\mathrm{E}\left[\mathrm{u}\left(\mathrm{H}_{\mathrm{i}} \mid \mathrm{y}^{\mu}\right)\right]$ has already been calculated. 
$\mathrm{E}\left[\mathrm{u}\left(\mathrm{H}_{\mathrm{i}} \mid \mathrm{y}^{\mu}\right)\right]$ is the expected utility (e.g. expected QALY or Cost) associated with the transition from all the stochastic subtrees Hi to state $y$, then at $\mathrm{G}$, the $\mathrm{E}[\mathrm{u}(\mathrm{G})]$ :

$$
\begin{gathered}
E[u(G)]=\left(\frac{v(y)+\sum_{i}\left(\mu_{i} E\left[u\left(H_{i} \ll y^{\kappa}\right)\right]\right.}{r(y)+\lambda}\right) \\
E[u(G)]=\left(\frac{v(y)+\mu \sum_{i}\left(p_{i} E\left[u\left(H_{i} \ll y^{\kappa}\right)\right]\right.}{r(y)+\lambda}\right)
\end{gathered}
$$

Here, $y^{k}$ denotes a sojourn of random duration $k$ in health state $y .0 \leq v(y) \leq 1$ is a quality of life or a cost value specified to health state $y$, and $0<\mathrm{r}(y) \leq 1$ is a patient's risk assessment factor to have treatment; $\mathrm{r}(\mathrm{y})$ could be a discount value on the associated cost of a health state $y \cdot r(y)+\lambda>0$, and the symbol « means reached by.

\section{Our Model I Updating on Hazen's Formula}

Let $\mathrm{u}\left(y^{\prime \prime}\right)$ is a utility associated at state $y$. To compute QALY or treatment Cost (the utility), of a health state $y$, and according Hazen's formula:

$$
u\left(y^{\text {Exponential }}\right)=\int_{0}^{\infty}\left(\int_{0}^{t} v(y) e^{-r(y)^{*} k} d k\right) * \lambda e^{-\lambda * t} d t \ldots \ldots \text { (6) }
$$

For the state y sojourn, we replacing the exponential distribution, that Hazen used, with the WAFT model, $f(\mathrm{t})=\left(\Phi^{\gamma} \lambda \gamma \mathrm{t}^{\gamma-1}\right) \exp \left(-\lambda \Phi^{\gamma} \mathrm{t}^{\gamma}\right)$. We set $\gamma$ to one in the WAFT distribution to keep WAFT having e.g. Markov preference summary property for rollback computations. To compute the utility using $\lambda \Phi e^{-\lambda \Phi t}$, we have then:

$$
u\left(y^{W A F T}\right)=\int_{0}^{\infty}\left(\int_{0}^{t} v(y) e^{-r(y)^{*} k} d k\right) * \lambda \phi e^{-\lambda \phi^{*} t} d t \ldots \ldots(7)
$$


In this formula, we have WAFT distribution behaving same as the exponential distribution, plus it has the patient's health condition too.

$$
\begin{gathered}
u\left(y^{\text {WAFT }}\right)=\int_{0}^{\infty}\left[\frac{v(y)}{r(y)}\left(1-e^{-r(y)^{* t}}\right)\right]^{*} \lambda \phi e^{-\lambda \phi^{*} t} d t \\
u\left(y^{\text {WAFT }}\right)=\frac{v(y)}{r(y)} \int_{0}^{\infty}\left(1-e^{-r(y)^{* t}}\right) * \lambda \phi e^{-\lambda \phi^{* t}} d t \\
u\left(y^{\mathrm{WAFT}}\right)=\mathrm{v}(\mathrm{y}) /(\mathrm{r}(\mathrm{y})+\lambda \Phi)------(8)
\end{gathered}
$$

And $r(y)+\lambda \Phi>0$

Let notation $\left[\begin{array}{l}\alpha \\ \beta\end{array} \mid(t)=\alpha+\beta t\right.$. , represents the affine function with slope $\alpha$ and intercept $\beta$, (see Hazen-Pellissier, 1996, page 794), Suppose $f$ and $g$ are functions, let the symbol $f_{o} z$ denote functional composition of $f$ and $z$, so that $\left(f_{o} z\right)(t)=f(z(t))$.

Fitting with semi-Markovian utility, and according to Hazen-Pellissier, 1996, and Hazen- Pellissier-Chang, 1996, our recursive formula, under WAFT model, of the expected utility of $\mathrm{G}$, will be:

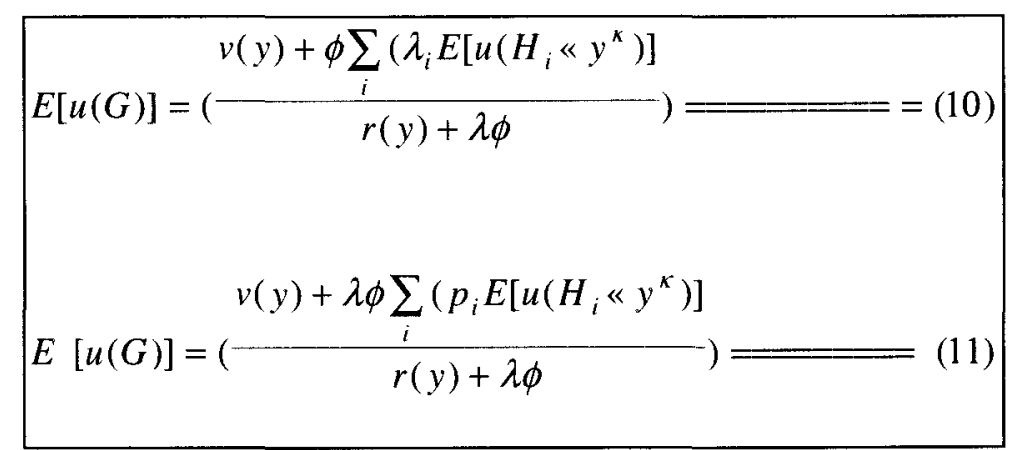

$$
r(y)+\lambda \Phi>0, \text { and } r(y)<>0
$$


Subtrees, $H_{i}$ are reached from state $y$ at competing rates, see figure (2). We also, supposed that no toll is associated with the transition from state $x$ to state $y$. Form (10) is used for a stochastic fork (arcs with rates); and form (11) is used for a chance fork (arcs with probabilities). $\sum \mu_{i}=\mu=1$, if there is chance fork reached by state $\mathrm{y}$ with immediate transition.

\section{Model I. Conclusion of the analytical model: "An Enhancement of Hazen's}

\section{Stochastic Tree Model Using Weibull Accelerated Failure Time Model for Medical}

Decision Making in Recurrent Diseases/Injuries"

Provided $r(y)+\lambda \Phi>0$ for formula $\# 8$ and $\# 10$, in the risk neutral case $r(y)=0$, we gain quality adjusted duration $u\left(y^{W A F T}\right)=v(y)^{*}(\lambda \Phi)^{-I}$. Focusing on Hazen and Pellissier model, there was a difficulty with the combination of memoryless utility and exponential duration that was: $u\left(y^{\text {Exponential }}\right)$ diverges to $+\infty$ when $r(y)+\lambda \leq 0$. So only moderately risk seeker preference $(-\lambda \leq \mathrm{r}(\mathrm{y}) \leq 0)$ may be portrayed (see Hazen and Pellissier, 1996, page

796). Our using of WAFT distribution, will cause $u\left(y^{W A F T}\right)$ diverges to $+\infty$ when $r(y)+\lambda \Phi$ $\leq 0$. Therefore more risk seeker preference $(-\lambda \Phi \leq \mathrm{r}(\mathrm{y}) \leq 0)$ may be portrayed when $\Phi>1$.

We introduce an extension to the analytical solution, stochastic tree modeling. In specific, the Weibull accelerated failure time model was utilized instead of an exponential distribution. This enhancement may increase the stochastic tree modeling technique's capabilities, by using different state sojourn distribution and by including a correct boundary difference between experimental treatment and the standard of care.

However, the stochastic tree model utilizing the Weibull accelerated failure time model should be restricted to have a memory less property for the rollback computation with respect to the semi-Markov requirements. 


\section{Multiobjective Simulation-Based Model for Medical Decision Making On Recurrent Disease Treatment (Model II)}

\section{Model II. Introduction}

A variety of methodologies have been employed for decision making related to the treatment of recurrent diseases/injury. This model presents an investigation of the use of simulation on the stochastic tree, with associated techniques related to ranking and selection, and multi-objectives decision analysis.

The enhanced model, in the first part of this study, was still restricted to have the preference summary- memory less property- for the rollback computation. Consequently, it was still limited to specific survival distributions. So, further work was required to add more capabilities and extension not only for the related limitations on the use of specific survival distributions, but also to add multi-performance measures approach.

For this second part of this research, we developed a simulation model that maintains multiple input parameters and multiple performance measures. In our study, the following research section describes our design of the simulation model that enhances and extends the use of stochastic tree, the application and details of how we use the simulator, multiple attribute utility theory, and statistical ranking and selection (R\&S) to select the best medical intervention configuration of $K>1$ possible configurations. 


\section{Model II. Background}

Many publications are related to stochastic models in medical decision making.

Some researchers concentrate their efforts on medical outcomes and others focus on minimizing the cost of treatment. Integration and contribution of these can be obtained and it would be added valuable information.

Traditional decision analysis is used to indicate decisions favoring good outcomes even if there is risk adjoining the decision. The value of each possible outcome of a decision, whether measured in costs and benefits or utility, is usually variable. As the number of treatment options and strategy choices has exploded and the cost of carrying out research has varied, the best treatment for all clinical situations cannot be determined by conducting randomized controlled trials only.

\section{Model II. Specifications and Design}

Most of the R\&S literature focuses on procedures that are designed for scalar performance measures (Bechhofer, 1995). However, some multivariate results do exist. Gupta (1979) describes procedures that are based on scalar functions of the mean and covariance matrix of the multivariate populations. Gupta's procedures reduce the multivariate performance measure problem to a scalar performance measure problem. Unlike the procedures described in Gupta, our method does not require the complicated step of estimating a covariance matrix. As a result, the $R \& S$ approaches (i.e. Law and Kelton, 2000) that we use are simpler to implement and belong to a class of procedures whose properties are better understood. 
In a medical setting, a different approach is often used: Convert project performance over multiple measures to a scalar measure using costs. Even though this type of "costing" or pricing out performance measures has many noticeable advantages, it has some disadvantages as well. For example, perfect cost data may not be available because of not enough resources. Moreover, it may be not easy to accurately attach a dollar value to intangibles (e.g., the quality of life) even if the resources are available.

In our simulation model, we utilize an approach, similar to Morrice et al. (Morrice 2001), to this problem: convert multiple performance measures to a scalar performance measure using multiple attribute utility (MAU) theory (Keeney and Raiffa 1976). MAU theory can be used with the costing approach and it can be used instead of a costing approach when good cost data are not available or when cost is not suitable as a measure of performance.

In this research, we extend Hazen's stochastic tree using discrete event simulation modeling, MAU theory, and statistical R\&S with indifference-zone approach of Law and Kelton, (2000), to select the best medical intervention configuration from a set of $K$ configurations when a medical intervention performance is determined by $n$ performance measures, $n>1$. In our model, we follow the same combination technique of Morrice et al. $(1998,1999)$ to link R\&S procedure with MAU theory.

We developed a simulation model that maintains multiple input parameters and multiple performance measures. This part of our research describes our design of the simulation model that enhances and extends the use of stochastic tree, the application and details of how we use the simulator, multiple attribute utility theory, and statistical 
ranking and selection $(R \& S)$ to select the best medical intervention configuration of $K>1$ possible configurations.

$\underline{\text { Figure } 2}$ Our General Simulation Model's Components

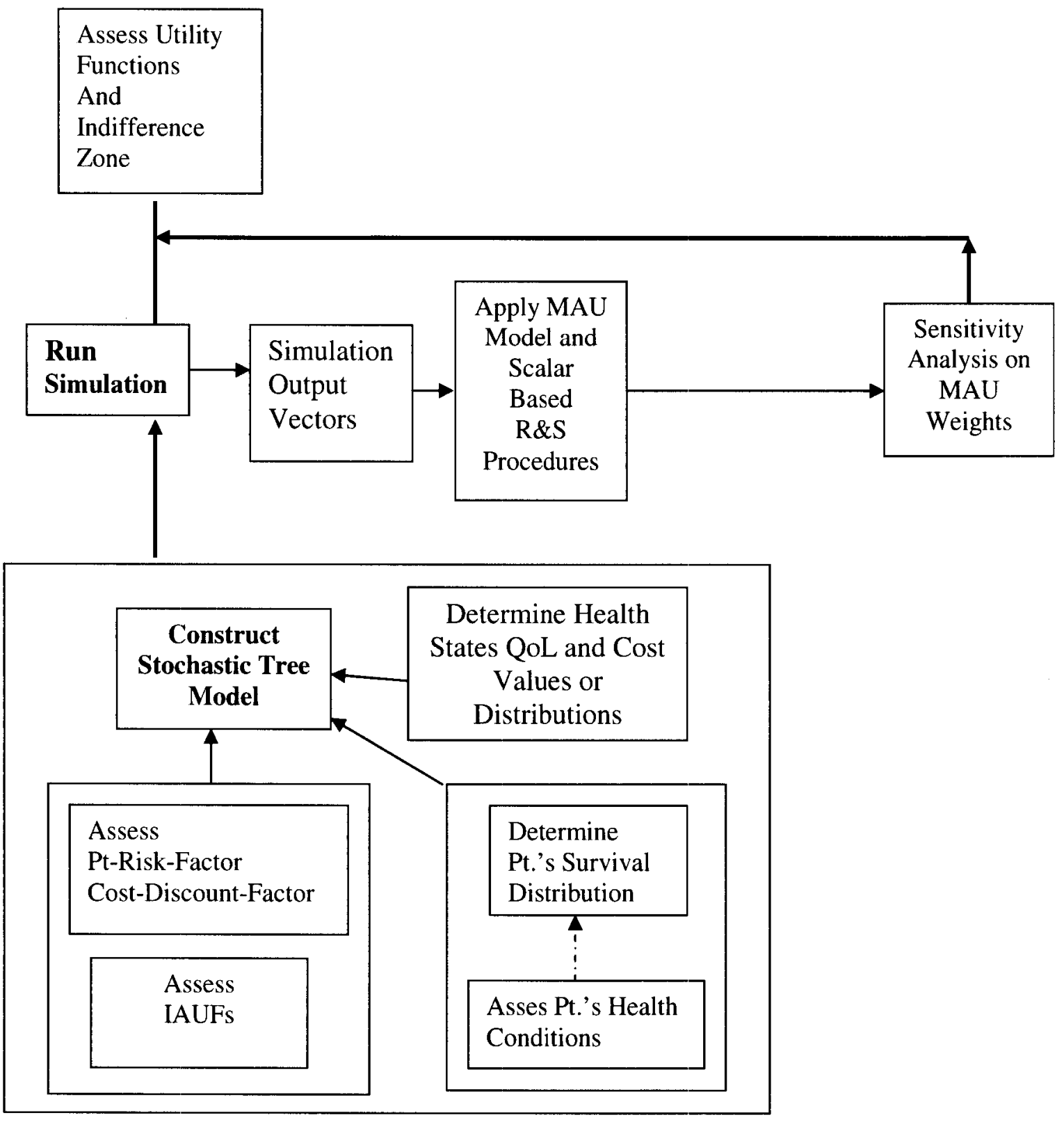




\section{Model II Definitions}

To determine to which type of experiment our simulation model belongs, the types of experiments should be briefed. There are three types of experiment: the first is Treatment Comparisons; its purpose is to compare several treatments of a factor (for example, suppose that one compares three diets and he/she would like to see if they are different in terms of effectiveness). The second is Variable Screening; (for example, suppose that one checks a large number of factors, but only a few are important, the experiment should identify the important, few, factors. The third is Response Surface Exploration; after important factors have been identified, their impact on the system is explored to optimize the response. Our Model is related to the first type of experiments.

Our Simulation output analysis computes measures of performance using the output mean, variance, confidence interval. Our model can work for comparing alternative designs in both (1) Simulations with a single model and different input data, and (2) Simulations with different models.

There are what is called terminating simulations, and non-terminating simulations. Our model is a terminating simulation. Terminating simulations, in which there is a natural event that specifies the length of each run; no useful information is obtained beyond termination; and termination time can be random. Non-terminating simulations, in which there is no natural event to specify the length of a run.

So, for the effect of initial conditions in terminating simulations, initial conditions generally affect measures of performance, and initial conditions should be chosen so that they are representative of the state of the system. Because our model is not a non- 
terminating simulation model, our interest does not lie in the steady-state performance measures.

Definition: One popular measure of treatment efficacy used in the medical decision making literature is mean quality-adjusted duration [Weinstein et al. 1980]. This measure is calculated by weighting the average duration spent in each particular health state by a quality rate proportional to the desirability of that state. Typically the Well state is assigned a quality factor of 1 , the dead state 0 , and other states intermediate values. The result for each treatment is a duration which, if spent entirely in the Well state, would be equivalent to receiving the treatment and all its consequences.

\section{Definition: A Stochastic Process is a family of random variables: $\{X(t), t<=\mathrm{T}\}$}

where $t$ is often interpreted as time; $X(t)$ is the state of the process at time $t$; $\mathrm{T}$ is the index set of the process.

- The process can be continuous or discrete. The state space of a stochastic process is the set of all possible values that $\mathrm{X}(\mathrm{t})$ can take on. The state space of the process is the set of all possible values that the random variables $\mathrm{X}(\mathrm{t})$ can assume.

- Stochastic processes can be classified according to these characteristics: (1) the state space may be discrete (finite or countable) or continuous. Accordingly, the stochastic process is called a "discrete-state process" or a "continuous-state process". Discrete-state processes are also called "chains". (2) The time parameter is another characteristics of stochastic processes; if the set of points on the time axis at which changes in $\mathrm{X}(\mathrm{t})$ may take place is finite or countable, the stochastic process is called a "discrete-time process". On the other hand, if X(t) may change its value anywhere within a finite or infinite interval of the time axis, the process 
is called a "continuous-time process". (3) The statistical dependency among the values of $\mathrm{X}(\mathrm{t})$ for different values of $\mathrm{t}$.

System Setting: Consider some type of treatment for a disease/injury and the modeling of the effects of that treatment. Suppose that the progression of the disease/injury following the treatment can be modeled as the progression of the patient through a series of states, enumerated as: $1,2, \ldots n$, where $n$ will be defined as the terminating state. Let

$S=$

Transition Probability $(\mathbf{I}, \mathrm{J})=$

Time in State $(\mathrm{I}, \mathrm{J})=$

$\mathrm{QoL}(\mathrm{I})=$

Quality of Life $=$
Number of States.

the probability that a transition will be made from State I to State J.

the distribution function representing the amount of time that the patient will spend in State I, given that a transition will be made to State J. Note that this will only be defined if Transition Probability (I, J) $>0$. (This will be modeled as an Arena Expression). the Quality of Life associated with State I. a time persistent variable representing the quality of life at a particular point in time.

Then Quality Adjusted Life Years is computed at the end of the simulation run (when the patient reaches the terminal state) as the integral over time of the Quality of Life. Each run of the model will yield a different value for QALYs, so 1000 runs will be 
made of the demonstration run. The demonstration run will have 5 states, where State 5 will be defined as the Terminal State, with the following transition:

$\begin{array}{cccccc}\text { State: } & 1 & 2 & 3 & 4 & 5 \\ 1 & - & 70 & 30 & 0 & 0 \\ 2 & 40 & - & 10 & 50 & 0 \\ 3 & 20 & 30 & - & 10 & 40 \\ 4 & 0 & 20 & 60 & - & 20 \\ 5 & - & - & - & - & -\end{array}$

\section{Model II. Inputs}

The inputs of the model are Survival Distributions matrix, Transition Rates matrix, Patient's Risk assessment factors, Decision Maker's Risk assessment factor, Cost Discount Factor, Quality of Life, Medical Intervention Costs, and Performance Measures Weights: (1) the Survival distributions matrix, $\mathrm{T}$, is a one-dimension matrix contains cells corresponding to patient's health states; each cell of the matrix may contain one value (median) or a survival distribution. A survival distribution, for a health state sojourn times, would be any survival distribution such as Exponential, Weibull, Weibull with accelerated failure time model, and Gamma, etc. (2) Transition Rates matrix (if any): due to moving from a state of life to another state; transition rates matrix would be a onedimension corresponding to patient's health states. (3) The Patient's Risk assessment factors (Patient $t_{\text {RiskF }}$ ): for patient's utility assessment, we use Hazen's pay off approach; this requires the physician to elicit from the patient his or her risk assessment. (4) 
Decision Maker's Risk assessment factor $\left(\mathrm{DM}_{\text {RiskFactor }}\right)$ : this requires the decision maker to determine his or her risk assessment. (5) Cost discount factor $\left(\right.$ Cost $\left._{\text {DiscFactor }}\right)$ : it is a factor to compute the treatment cost as a performance measure (6) Quality of life, QoL, it is a one-dimension matrix contains QoL values for patient's health states; each cell may contain a value or a distribution. (7) Medical cost of a health state, $\mathrm{C}$, is a one-dimension or two-dimension matrix of the health states of life; each cell could contain one value or a distribution. (8) The importance level values or the weights for the performance measures, $l_{1}$ and $l_{2}$. The decision maker utility function; $\Sigma l_{\mathrm{i}}=1$, if Ui are utility independent, $\Sigma l_{\mathrm{i}}<>1$, if $\mathrm{Ui}$ are not utility independent.

At the beginning of each simulation, some inputs are initials values. The system outputs may change by these values; for example, performance measures weights, $l_{\mathrm{i}}$, that represent the DM preferences, and also the 1st Health State that the patient begins with.

\section{Model II. Computations}

For each replication the simulation system will compute seven formulas:

1. The first formula is to compute the Individual's Attribute Utility Factor of Risk $\left(\mathrm{IAUF}_{1}\right)$, and $\mathrm{IAUF}_{1}=1-\exp \left(-\right.$ StayTime $*$ Patient $\left._{\text {RiskF }}\right)$. This is a common formula for a risk avoider person. The formula fits with an individual faces a medical intervention that may have some level of risk.

2. The second formula is to compute the Individual's Attribute Utility Factor of Cost $\left(\mathrm{IAUF}_{2}\right)$, and $\mathrm{IAUF}_{2}=1-\exp \left(-\right.$ StayTime $^{*}$ Cost $\left._{\text {DiscFactor }}\right)$. This is a common formula for a risk avoider person, who faces a costly medical intervention.

3. Quality Adjusted Life Years for each state of life, $\mathrm{QALY}=\mathrm{QoL}^{*} \mathrm{IAUF}_{1} * \mathrm{~T}$ 
4. Adjusted Cost for each state of life, COST $=\mathrm{C} * \mathrm{IAUF} 2 *$ StayTime

5. Decision's maker utility function on QALY, PerfMeasure ${ }_{1}$, is a risk avoider utility function; its formula is: $\mathrm{U}_{1}=1-\exp \left(-\mathrm{QALY}^{*} \mathrm{DM}_{\text {RiskFactor }}\right)$; and $0 \leq \mathrm{U}_{1} \leq 1$

6. Decision's maker utility function on Cost, PerfMeasure ${ }_{1}$, is a risk avoider utility function; its formula is: $U_{2}=1$ - $\exp \left(-X *\right.$ Cost $\left._{\text {DiscFactor }}\right)$; hence $X=1 /($ Cost in $\$ 10,000 \mathrm{~s})$, Cost $<>0$, and $0 \leq \mathrm{U}_{2} \leq 1$

7. Expected decision maker's utility function can be:

$\mathrm{E}(\mathrm{U})=\mathrm{w}_{1} * \mathrm{u}_{1}+\mathrm{w}_{2} * \mathrm{u}_{2}+\left(1-\mathrm{w}_{1}-\mathrm{w}_{2}\right) * \mathrm{u}_{1} * \mathrm{u}_{2}$, Or

$\mathrm{E}(\mathrm{U})=\mathrm{w}_{1} * \mathrm{u}_{1}+\mathrm{w}_{2} * \mathrm{u}_{2}$ if the $\mathrm{U} 1$ and $\mathrm{U} 2$ are utility independent.

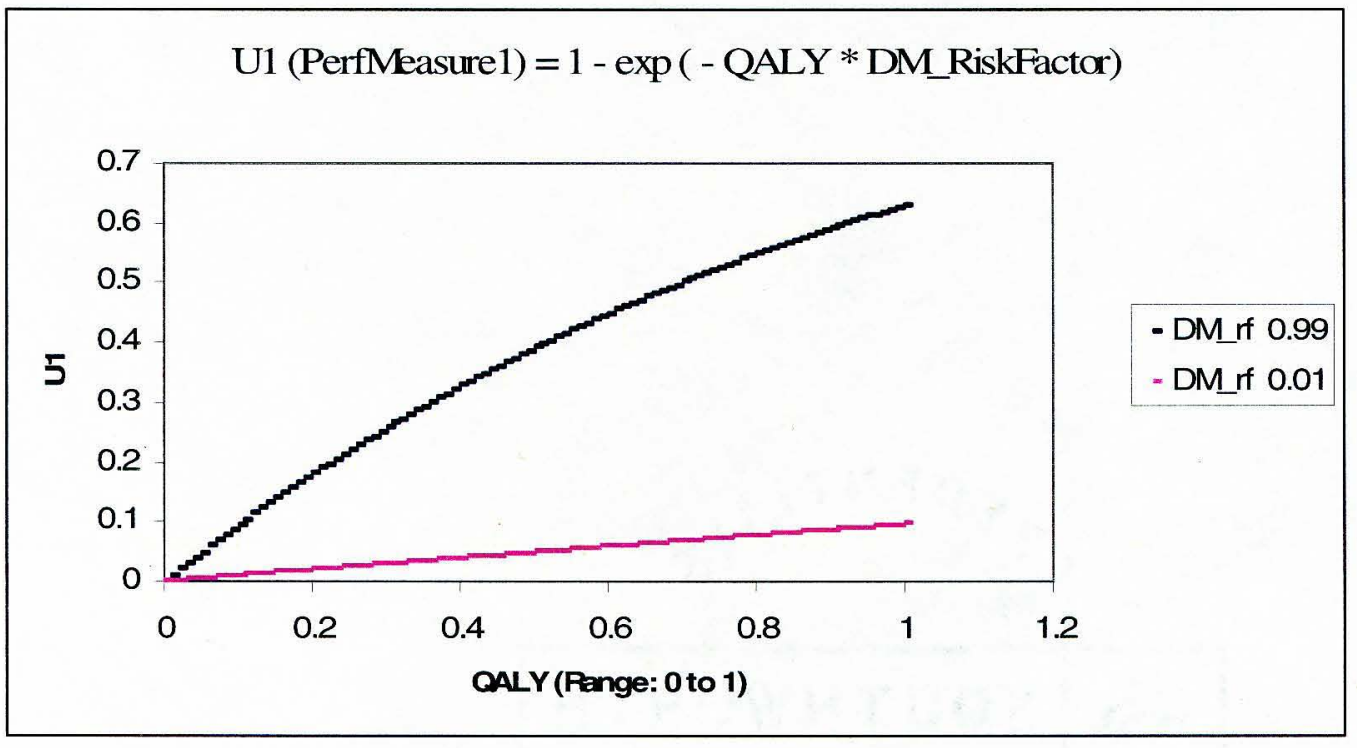

Figure 3: Risk avoider utility function on performance measure, QALY 


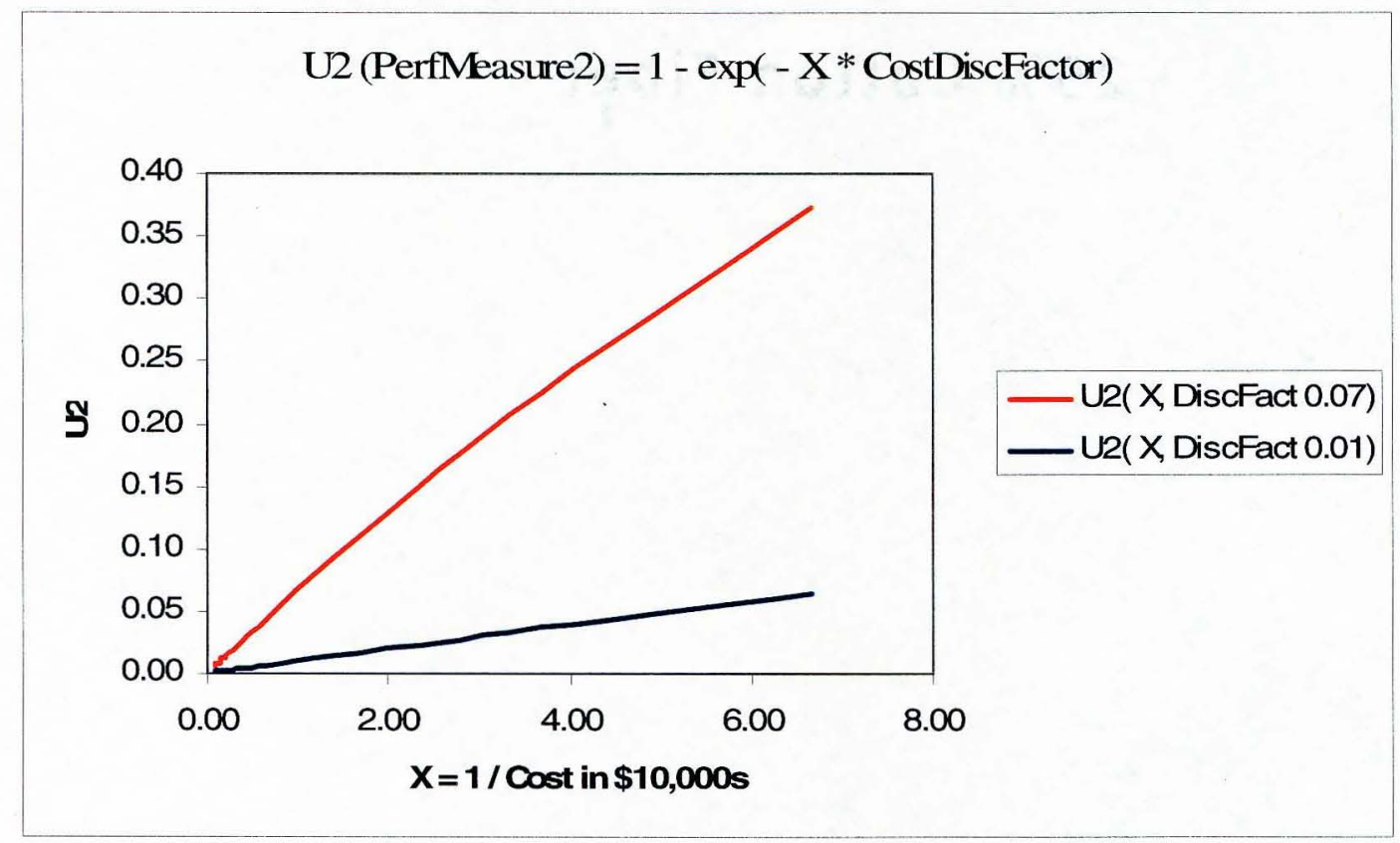

Figure 4: Risk avoider utility function on performance measure, Treatment Cost

\section{Model II. Outputs}

At the end of each simulation, with $n$ replications, the simulation system will output five performance measures:

1. Mean of the first performance measure; which is QALY of all health states.

2. Mean of the second performance measure; which is Cost of all health states.

3. Mean of the utility function of the first performance measure on QALY, U1.

4. Mean of the utility function of the second performance measure on Costs, U2.

5. Expected Utility function, $E(U)$, for the decision maker's, on $U 1$, and $U 2$ 


\section{Risk Utility Assessment Used In Model II}

Hazen-Pellissier, (1996), presented von Neumann-Morgenstern utility functions over stochastic trees that allow easy rollback computation. One such utility function is a risk-sensitive extension of QALY that allows different constant coefficients associated with risk aversion across health states. Continuous-risk utility assessment protocol, as described by Hazen (1997), differs from standard preference assessment protocols by offering choices between alternatives in which risks are present in continuous time. For medical applications, "continuous-risk utility assessment scenarios are both more realistic and more familiar than other simpler but more artificial standard gamble approaches". (Hazen 1997) We use/recommend the same protocol.

To understand how continuous-risk utility assessment works consider the scenario in Figure \# , used to assess a subject's risk attitude about future time spent in morbidity functional class IV for osteoarthritis of the hip. The subject is asked to determine what compensating immediate mortality risk $\mathrm{p}$ he is willing to gain to decrease his mortality rate from the high rate $\mu_{1}$ to a lower rate $\mu_{0}$. The subject's response determined his or her coefficient of risk aversion for health state: "morbidity functional class IV".
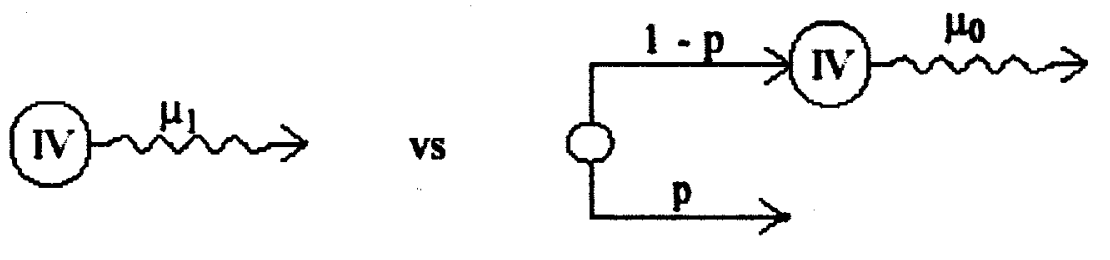

Figure 5 Continuous-risk assessment approach, by Hazen 1995.

With this continuous-risk assessment approach, a patient must determine what chance $p$ at immediate death he/she is willing to accept to improve his future mortality rate from $\mu_{1}$ to $\mu_{0}$. 


\section{Model II Design on Arena Software}

Arena is a MS-Windows application, and all the typical Windows features and operations are available. Also, Arena is fully compatible with common MS-Windows software, such as MS-Word, and MS-Excel. In Arena models, the basic building blocks are called modules. These modules are flowchart and data objects that define the process to be simulated and are selected from panels in the project Bar. Flowchart modules describe the dynamic processes in the model. Flowchart can be as nodes or health states through which entities flow, or where entities begin or leave the model.

Flowchart modules are usually connected to each other by somehow. In the Basic Process panel, the kinds of flowchart modules accessible are several such as Create, Dispose, Process, Decide, Assign, and Record. Each type of flowchart module has a distinguishing shape.

Data modules define the characteristics of diverse process elements, such as entities, resources, and queues. In addition, data modules can set up variables and expressions and other types of numerical values that relate to the model. Icons for data modules, in what is called the Project Bar, look like little spreadsheet figures. The Basic Process panel's data models are several such as Entity, Queue, Resource, and Variable. Entities don't flow through data modules. Flowchart and Data modules are related to each other by user defined names for objects (e.g. Variables) that they have in common.

On the following three pages, our general simulation model is displayed on Arena; and six illustration figures are shown for the model design and setting. 


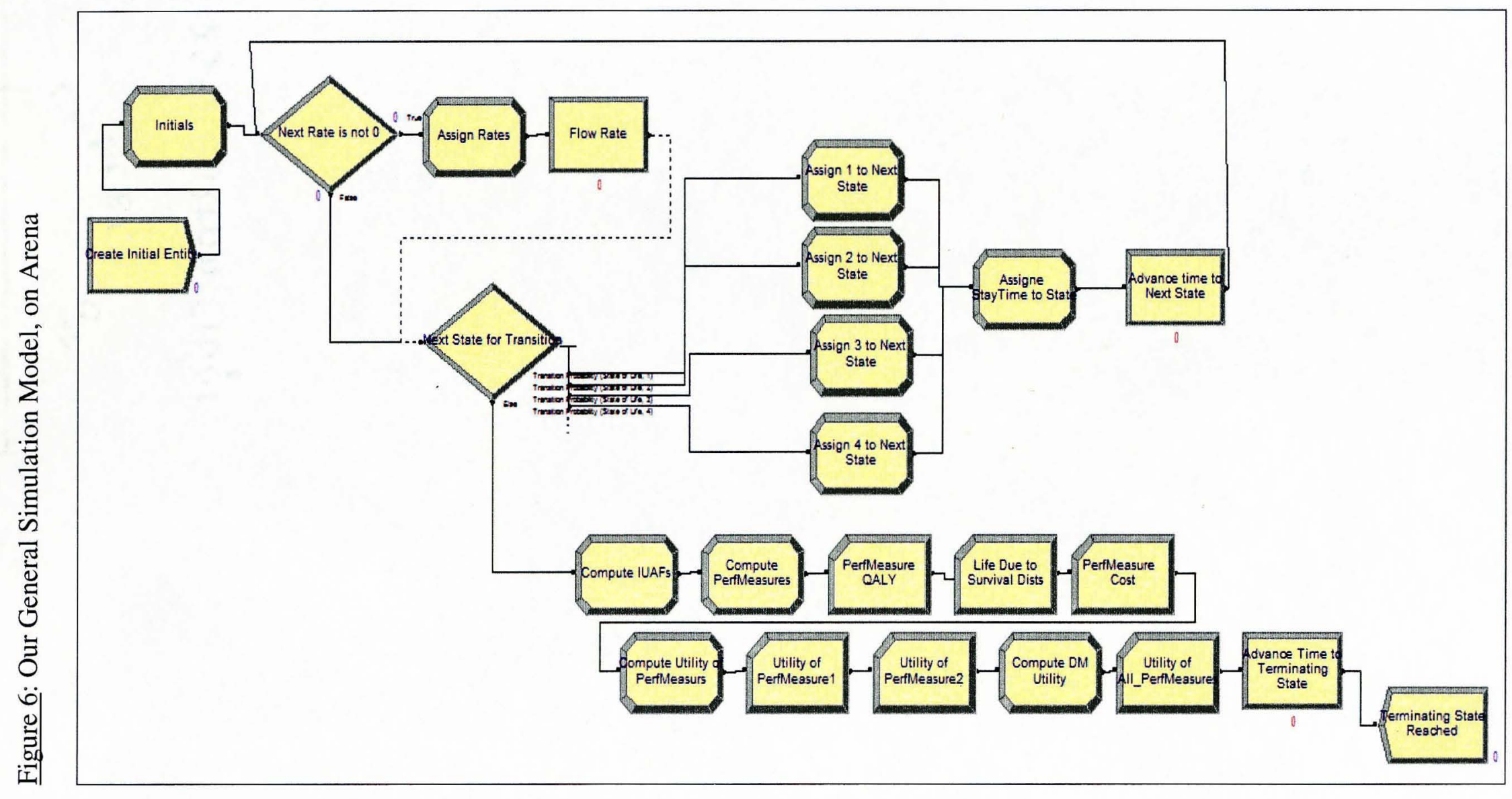




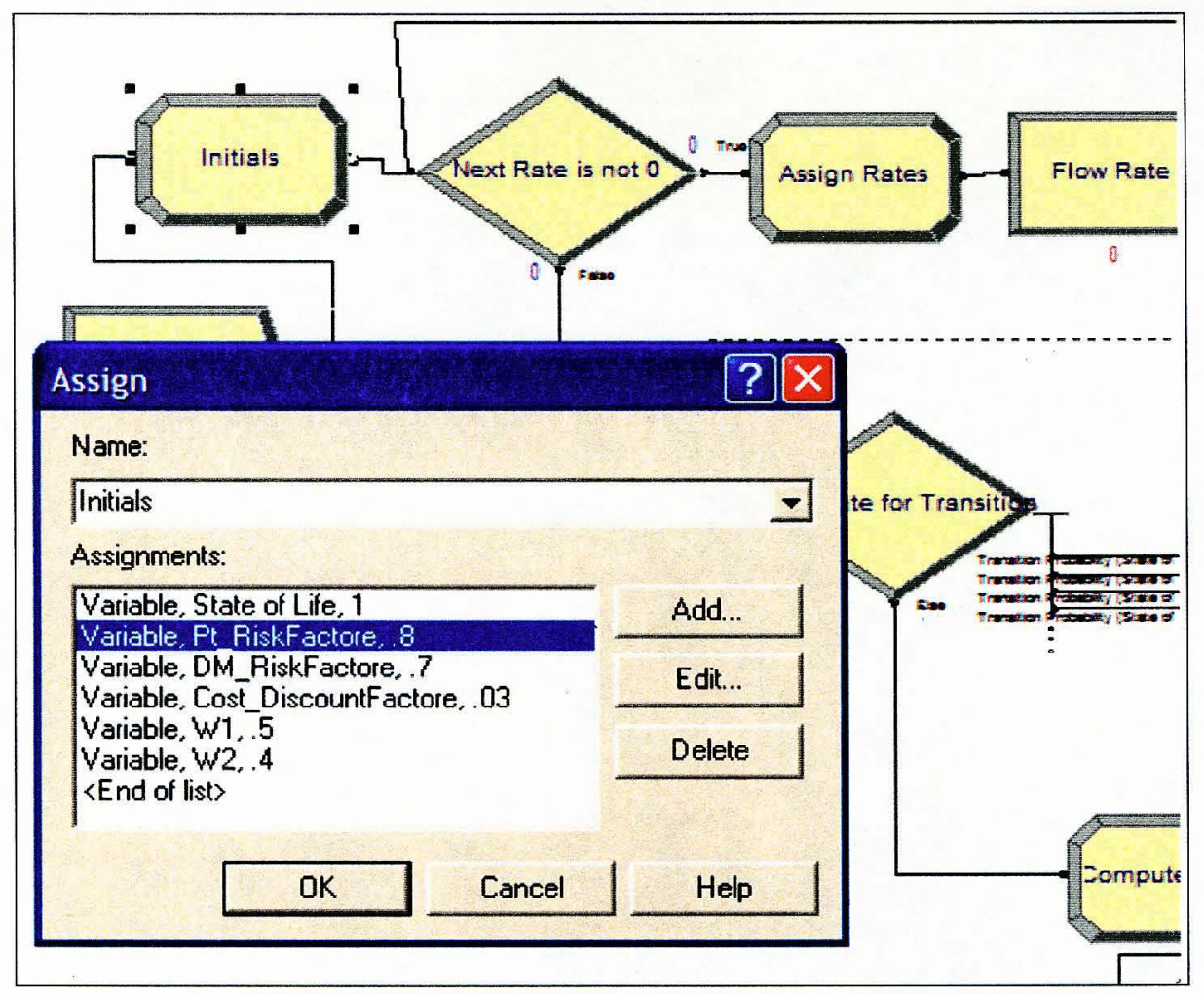

Figure 7: Our General Simulation Model Initials

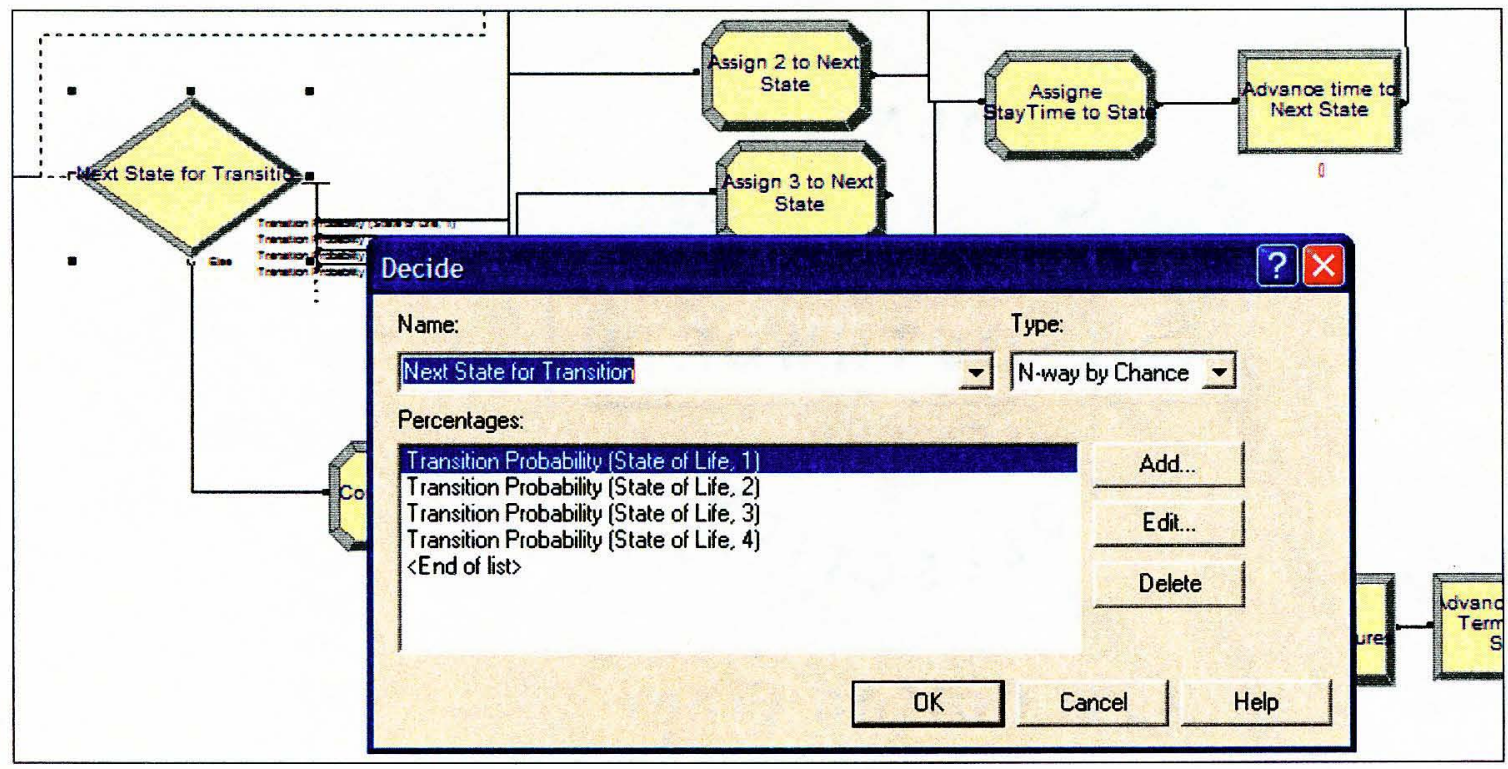

Figure 8: Our General Simulation Model Transition Probabilities Settings 


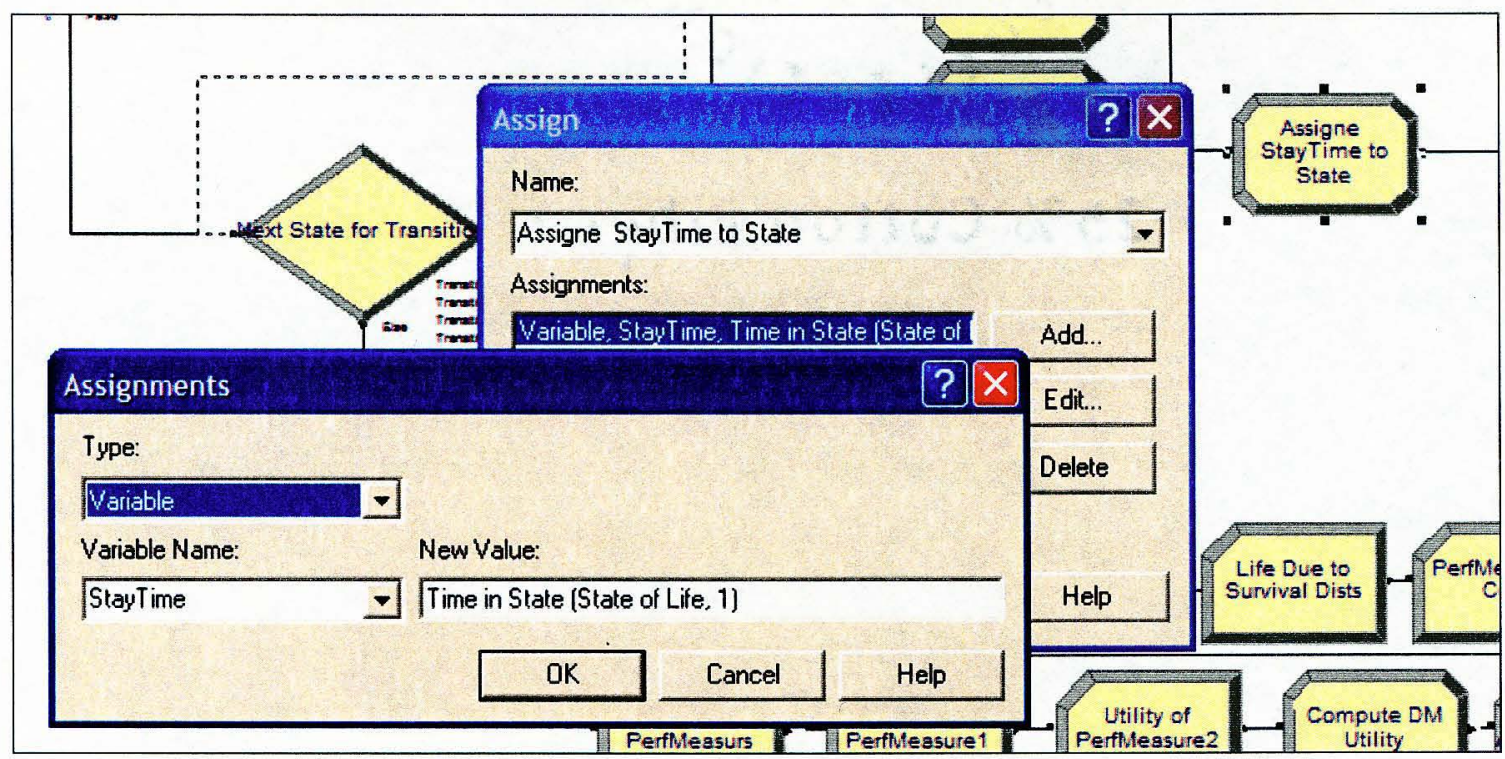

Figure 9: Our General Simulation Model Stay time Assignments

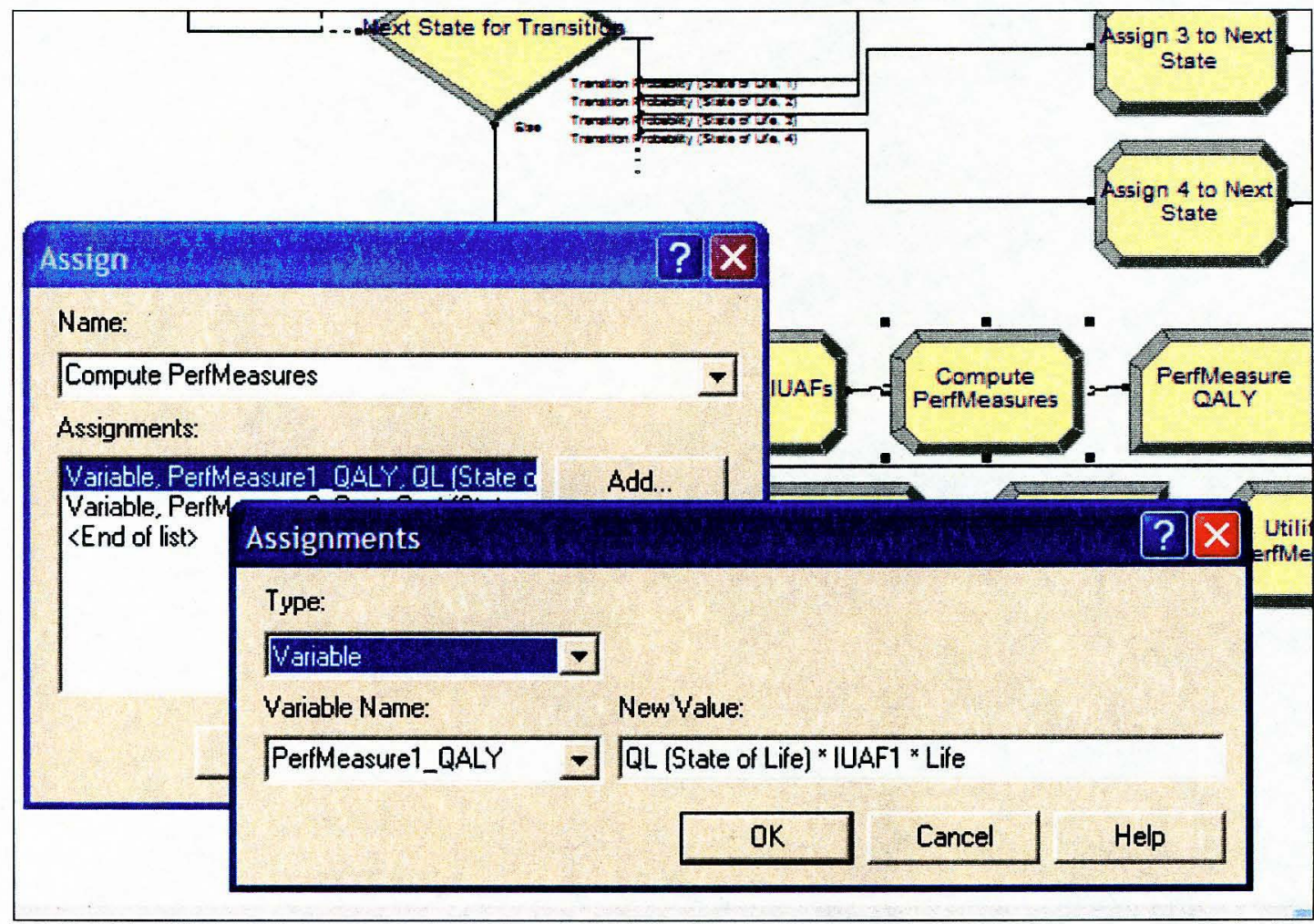

Figure 10: Our General Simulation Model Performance Measures Computing 


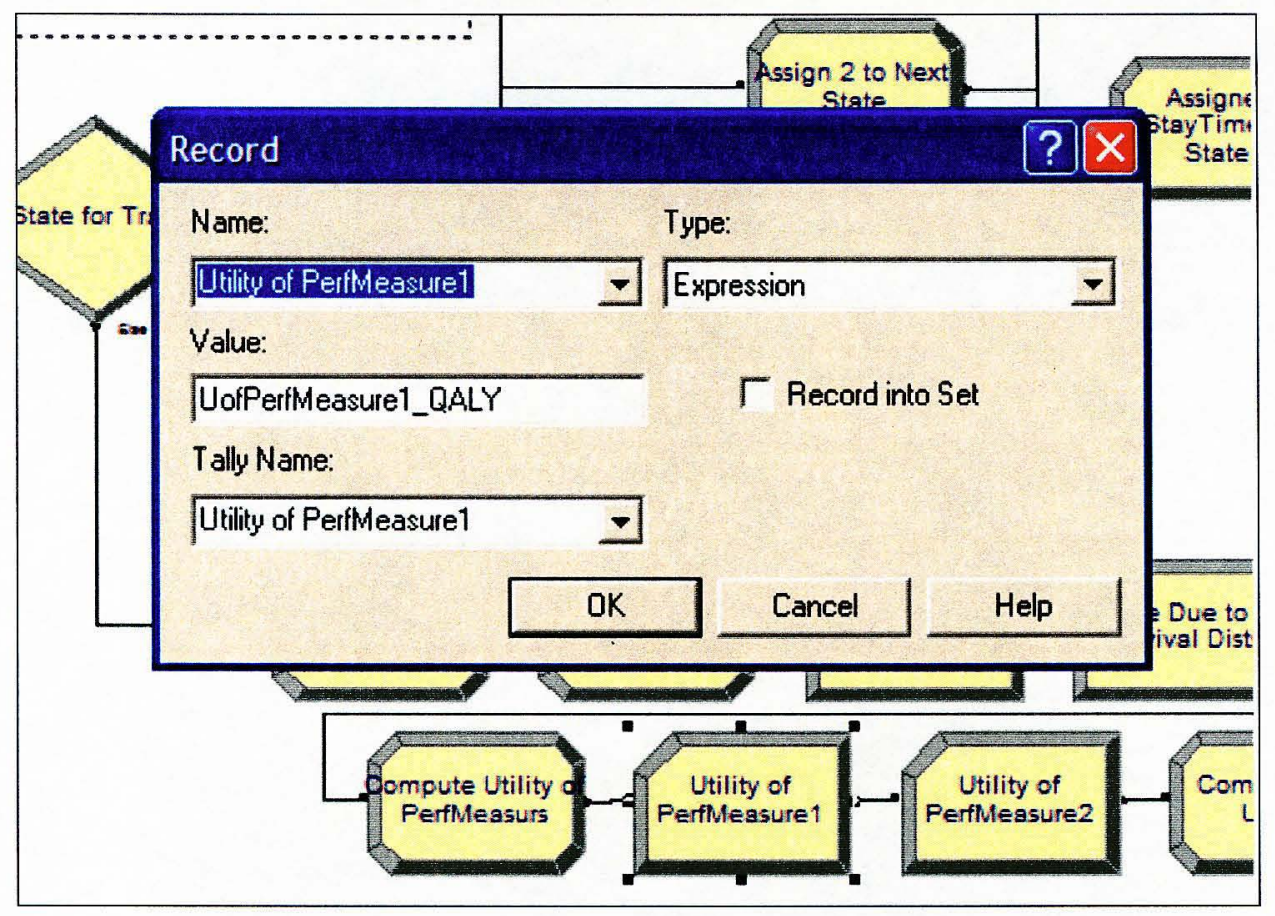

Figure 11: Our General Simulation Model Performance Measures Recording

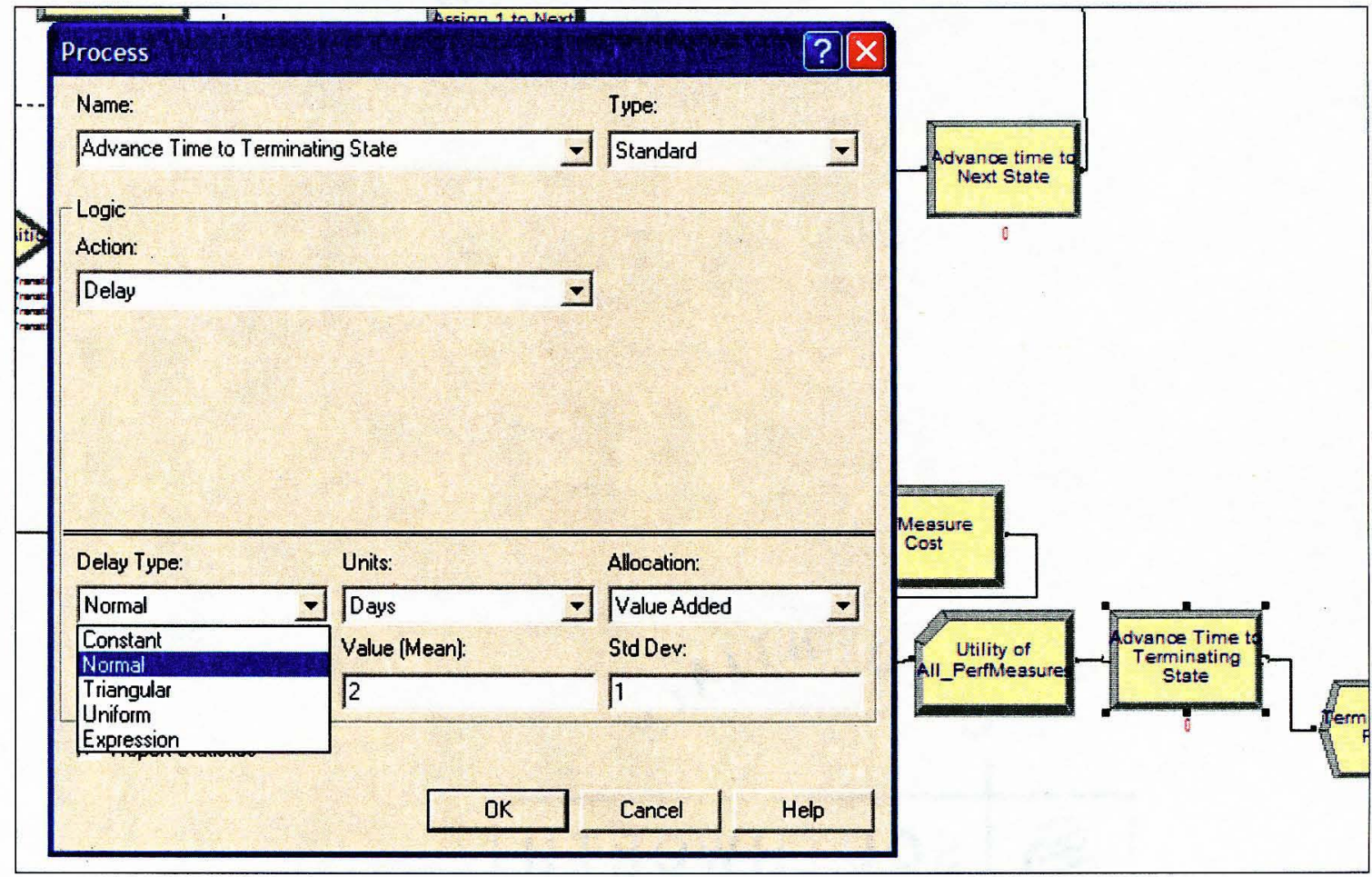

Figure 12: Our General Simulation Model Advancing Time 


\section{Ranking and Selection (R\&S) Experimental Settings}

We employed the ranking and selection procedure of. The goal of this procedure is to make a statement of the best system; where, "best" refers to the system with the maximum performance measure of interest for ith alternative treatment and $\mathrm{j}^{\text {th }}$ replication.

The general approach involves: denoting correct selection with the notation CS. We want $P(C S) \geq P^{*}$ provided that $\mu i-\mu j \geq d^{*}$, where $d^{*}$ defines an indifference amount. For differences smaller than $\mathrm{d}^{*}$ the choice may not be significant. The procedure is described on pages 30 and 31 . We need not assume anything about the variances of $\mathrm{Xij}$, as it is assumed that they are normally distributed. The procedure is, however, reasonably robust against departures of this assumption.

Multi-Attribute Utility theory, MAU, (Keeney and Raiffa 1992, Clemen 1991):

MAU is one of the major analytical tools of decision analysis. "A MAU analysis of alternatives (in our example, medical interventions) explicitly identifies the measures that are used to evaluate the alternatives, and helps to identify those alternatives that perform well on a majority of these measures, with a special emphasis on the measures that are considered to be relatively more important" (Morrice et al, 1998, page 720).

Our simulation model can run using the decision maker's preferences with the MAU additive or multiplicative model, In our examples we used two performance measures, which are $\mathrm{U}_{1}(\mathrm{QALY})$ and $\mathrm{U}_{2}$ (Treatment cost).

In MAU theory, the additive model is used if the $\mathrm{U}_{1}$ and $\mathrm{U}_{2}$ are utility independent. $\mathrm{E}(\mathrm{U})$ $=l_{1} * \mathrm{U}_{1}+l_{2} * \mathrm{U}_{2}$, where $0 \leq l i \leq 1 ; \quad \Sigma l i=1$. And, the model multiplicative model is used 
if the $\mathrm{U} 1$ and $\mathrm{U} 2$ are not utility independent. $\mathrm{E}(\mathrm{U})=l_{1} * \mathrm{U}_{1}+l_{2} * \mathrm{U}_{2}+\left(1-l_{1^{-}} l_{2}\right) * \mathrm{U}_{1} * \mathrm{U}_{2}$ ,where, $0 \leq l i \leq 1 ; \quad \Sigma l i<>l$

Supposing $R T i$ is the decision maker's assessed risk tolerance and $A i$ and $B i$ are scaling constants for measure $i$. a popular form is used in our model (see Clemen 1991, p. 379).The form is single-attribute utility function:

$$
u_{i}\left(x_{i}\right)=A_{i}-B_{i} e^{\left(x_{i} R T_{i}\right)}
$$

Settings: Assume that there are $\mathrm{K}>2$ medical intervention, with different configurations. For

$$
1 \leq k \leq K, \text { let } X_{k}=\left(X_{k 1}, X_{k 2}, \ldots, X_{k n}\right)
$$

denote a vector of random variables representing the performance measures for configuration $\mathrm{k}$. Let $\mathrm{E}[\mathrm{u}(\mathrm{Xk})]$ denote the expected utility (unknown) for configuration $\mathrm{k}$ and let

$$
\mathrm{E}\left[u\left(X_{|1|}\right)\right] \leq \mathrm{E}\left[u\left(X_{[2 \mid}\right)\right] \leq \cdots \leq \mathrm{E}\left[u\left(X_{|K|}\right)\right]
$$

This denotes the ordered expected utility values. The goal is to select the project configuration with the largest expected utility $\mathrm{E}\left[\mathrm{u}\left(\mathrm{X}_{[\mathrm{k}]}\right)\right]$. If the $\mathrm{R} \& \mathrm{~S}$ procedure accurately identifies the configuration with the largest expected utility, a "correct selection" (CS) is made.

In the two-stage $\mathrm{R} \& \mathrm{~S}$ procedure, such as Law and Kelron, guarantees that the probability of a correct selection, $\mathrm{P}\{\mathrm{CS}\} \geq P^{*}$, whenever: $\mathrm{E}\left[\mathrm{u}\left(\mathrm{X}_{[\mathrm{k}]}\right)\right]-\mathrm{E}\left[\mathrm{u}\left(\mathrm{X}_{[\mathrm{k}-1]}\right)\right]=\delta$ $\mathrm{P}^{*}$ is specified by the decision maker, and $\delta$ is a practical significance difference between system $X_{[k]}$ and $X_{[k-1]}$. In practice, the selection of $\delta$ depends on the decision of the decision maker. 
Determining $\delta$ Value: Suppose that there is a performance measure $\mathrm{X}$ on two systems, $\mathrm{X}_{[\mathrm{k}]}$ and $\mathrm{X}_{[\mathrm{k}-\mathrm{l}]}$, and $\delta$ represents the accepted difference, needed by the decision maker to distinguish between the two systems. $\delta$ is computed by $\delta=l_{l} . \delta_{l}+l_{2} . \delta_{2}$ where $\delta i$ represents a value computed by $\delta_{i}=\mathrm{E}\left[\mathrm{u}\left(\mathrm{X}_{[\mathrm{k}]}\right)\right]-\mathrm{E}\left[\mathrm{u}\left(\mathrm{X}_{[\mathrm{k}-1]}\right)\right]$, and $l_{i}$ represents the level of importance, assigned by the decision maker to each performance measure, $\Sigma l_{i} \leq 1$ (Butler and Morrice, 1998 \& 2001).

For example, suppose that there are two performance measures, Cost and QALY, measured for two systems. For the system number $k$ and the system number k-1, $\delta_{1}=\mathrm{E}\left[\mathrm{u}\left(\operatorname{Cost}_{[\mathrm{k}]}\right)\right]-\mathrm{E}\left[\mathrm{u}\left(\operatorname{Cost}_{[\mathrm{k}-1]}\right)\right]$, and $\delta_{2}=\mathrm{E}\left[\mathrm{u}\left(\mathrm{QALY}_{[\mathrm{k}]}\right)\right]-\mathrm{E}\left[\mathrm{u}\left(\mathrm{QALY} \mathrm{Y}_{[\mathrm{k}-1]}\right)\right]$. Suppose $\delta_{1}=0.007, \delta_{2}=0.018$ and the decision maker assigned the performance measure "Cost" a higher level of importance $l_{l}=0.65$, and the performance measure "QALY" a level of importance $l_{2}=0.35$. Then $\delta=0.65 * .007+0.35 * 0.018=0.011$

In this study, we applied our simulation model on an example that compared three treatment systems related to breast cancer related treatments. We used expected performance measures on Cost and QALY.

To establish an indifference zone for $\delta i$, its recommended to assess $\delta_{i}$ in the units of the performance measure and then converting to a number on the utility, $(0,1)$, scale. We will construct an indifference-zone-preference-zone diagram on the performance measure, (QALY, and Cost) by using the same procedure utilizing certainty equivalents on the single-attribute utility functions.

Having: $\mathrm{E}\left[u_{1}\left(X_{[K] l}\right)\right]=u_{I}\left(C E_{[K] l}\right)$

The indifference zone is defined by: $u_{l}\left(C E_{[K] l}\right)-u_{I}\left(C E_{[K-I] l}\right)>=\delta_{I}$ 
To establish the indifference-preference-zone on the original performance measure: "one can invert $u_{1}\left(\mathrm{CE}_{[\mathrm{K}] 1}\right)$ and $u_{1}\left(\mathrm{CE}_{[\mathrm{K}-1] 1}\right)$ and establish an indifference zone based on $\mathrm{CE}_{[\mathrm{K}] 1}$ and $\mathrm{CE}_{[\mathrm{K}-1] 1}$. The curve that divides the indifference zone from the preference zone is constructed by setting: $\mathrm{u}_{1}\left(\mathrm{CE}_{[\mathrm{K}] 1}\right)-\mathrm{u}_{1}\left(\mathrm{CE}_{[\mathrm{K}-1] 1}\right)=\delta_{1}$ and solving for $\mathrm{CE}_{[\mathrm{K}] 1}$. The resultant expression for the curve dividing the indifference zone from the preference zone is" (Morrice, 2001)

$$
C E_{|K| 1}=C E_{|K-1| 1}+R T_{1} \ln \left\{-\left(\frac{\delta_{1}^{*}}{B_{1}}\right) e^{\frac{-\sigma_{K-1 \mid 1}}{K T_{1}}}\right\}
$$

Where, $R T$ is the risk factor, and $B$ is constant, we will sit it to 1 to fit with the risk avoider utility function, (Morrice, 2001): $u_{i}\left(x_{i}\right)=1-e^{-x i R T i}$

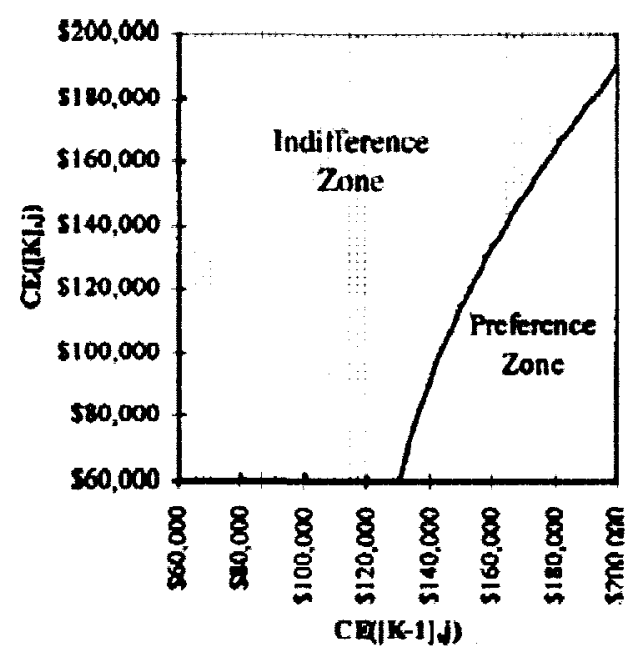

Figure 13 indifference-zone and preference-zone diagram. It is corresponding to $\delta_{1}$ equal to 0.2 , for the cost-utility function. This figure is taken from (Morrice, 2001). 
Sensitivity Analysis: Most applications of multiattribute utility theory focus on a series of sensitivity analyses to set up a sense of the robustness of the recommendations generated by the MAU model (Dyer et al. 1998). One common technique is to determine the impact of varying performance measure's weights, which are assessed by the decision maker, or the patient's risk factors, which are assessed by the decision maker, of the MAU model. Often this analysis is performed by varying one weight/factor at a time while the ratios among the other weights/factors are held constant. (Morrice et al. 1999). We used this approach to study patient's risk factors sensitivity for the breast cancer treatments application discussed in this research. Though, varying one patient's risk factors at a time can be misleading because it pays no attention to the potential interaction that can result from concurrent manipulations of multiple patient's risk factors (Butler et al. 1997). This matter can be eased by evaluating all probable combinations of weights/factors via Monte Carlo simulation (Butler et al. 1997). First, in randomly generating weights/factors for $\mathrm{k}$ attributes is to select $\mathrm{k}-1$ independent random numbers from a uniform distribution on $(0,1)$. Second, is to rank these numbers. We were able to use this information in a Monte Carlo simulation sensitivity analysis of the patient's risk factors. 


\subsubsection{Conclusion of the Model (II)}

Indeed, there are a number of issues that must be recognized as important for the success of our approach. Because we use a pay off model this requires elicitation by decision maker of both a utility function for each measure as well as a "weight," or indication of relative importance. Techniques for handling these types of elicitations are available (e.g. Clemen 1991 and Keeney and Raiffa 1976). And for patient's risk assessment, we recommend what is called continues risk assessment (Hazen 1995, Hazen-Chang, 1996).

The idea for the methodology outlined, in our simulation model, model II, is based on interactions of enhanced medical stochastic model, for evaluating multi treatment outcomes, with real decision makers whose decision inputs are the results of simulation experiments. 


\section{CHAPTER IV}

\section{RESULTS}

\section{An enhancement of the stochastic tree model for medical decision making}

Stroke places a huge burden on society in terms of premature death, disability, and costs of care. Increasingly, the cost-effectiveness of new interventions needs to be demonstrated before their widespread implementation. Clinical trials are unable to measure the long-term impact of such new interventions in stroke care, and a modeling approach is necessary. The Stroke Outcome Model has been developed using enhanced model of Hazen stochastic tree as a flexible tool for this purpose. Our model is used to undertake economic analyses of antiplatelet therapy for the prevention of recurrent strokes, and of stroke unit care and thrombolytic therapy in acute stroke.

CDC- Stroke Facts: Stroke is the third leading cause of death after heart disease and cancer and a leading cause of serious, long-term disability. In 2002, stroke killed 162,672 people ( $61 \%$ of them women), accounting for about 1 of every 15 deaths. The death rate was 56 per 100,000 populations. Stroke death rates are substantially higher for African Americans than for whites (2002 rates per 100,000 population: 82 for black men, 72 for black women, 54 for white men, and 53 for white women). For other racial and ethnic groups, 2002 stroke death rates per 100,000 population were 48 for Asians/Pacific Islanders, 41 for Hispanics, and 37 for American Indians/Alaska Natives. Approximately 
$50 \%$ of stroke deaths occur before the person reaches the hospital. Each year, about 700,000 people suffer a stroke (about 500,000 first attacks and 200,000 recurrent attacks). From the early 1970 s to the early 1990 s, the estimated number of non institutionalized stroke survivors increased from 1.5 million to 2.4 million. Medicare spent $\$ 3.6$ billion in 1998 on stroke survivors discharged from short-stay hospitals.

Michael Chambers et al, (2002), developed decision-analytic model that represents the management of acute stroke and long-term care and prevention of recurrence for stroke survivors. The latter consists of semi-Markov state-transition processes, with health states defined by therapy, disability, and occurrence of further stroke. He concluded that development of this model highlights the need for improved information on prognosis and resources used by stroke survivors and the importance of differentiating between economically distinct end points such as death, disabled survival and non-disabled survival, which may be combined as outcomes in clinical trials.

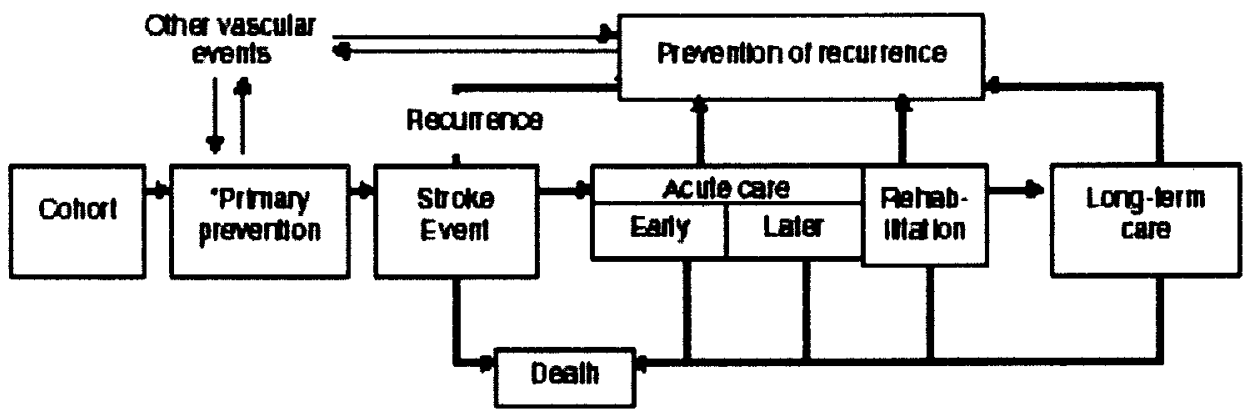

Figure 14: Schematic overview of Stroke Outcome Model.

This figure is taken from Michael Chambers et al (2002). 
Michael Chambers et al (2002) developed a long term care/prevention of recurrence module too; but their long term model does not compute Stroke outcomes.

Hazen on 1992 introduced a continuous-time analog of the Markov-cycle tree, the stochastic tree, which combines features of decision trees [Raiffa 1968] and stochastic process transition diagrams. Stochastic tree diagrams not only can depict continuously distributed temporal uncertainties, but, like decision trees, can be rolled back to determine optimal decisions.

We introduce a possible extension to the analytical solution, stochastic tree modeling. In specific, the Weibull accelerated failure time model was utilized instead of an exponential distribution. This enhancement may increase the stochastic tree modeling technique's capabilities, by using different state sojourn distribution and by including a correct boundary difference between experimental treatment and the standard of care.

\section{Example I: the Enhanced Stochastic Tree Model to Compute QALY for Stroke}

To calculated the QALY using the WAFT formula, on Chapter 3, we considered the same stroke model of Hazen, on figure 4, with the same probabilities and rates: $\mathrm{ms}=0.05$, me $=0.065, \mathrm{Pe}=0.38, \mathrm{~m} 0=0.0111, \mathrm{~Pb}=0.6667$; the quality adjustments: $\mathrm{v}$ (well) $=1.0$, and $\mathrm{v}($ PostBigStrok $)=0.2, \mathrm{v}($ PostSmallStrok $)=0.8$. In addition, we set for WAFT the initial values of: Patient's conditions factor $\Phi=0.7, \lambda .=0.009$, and Patient's Risk Assessment factor $\mathrm{r}(\mathrm{y})$ to 0.12 . 


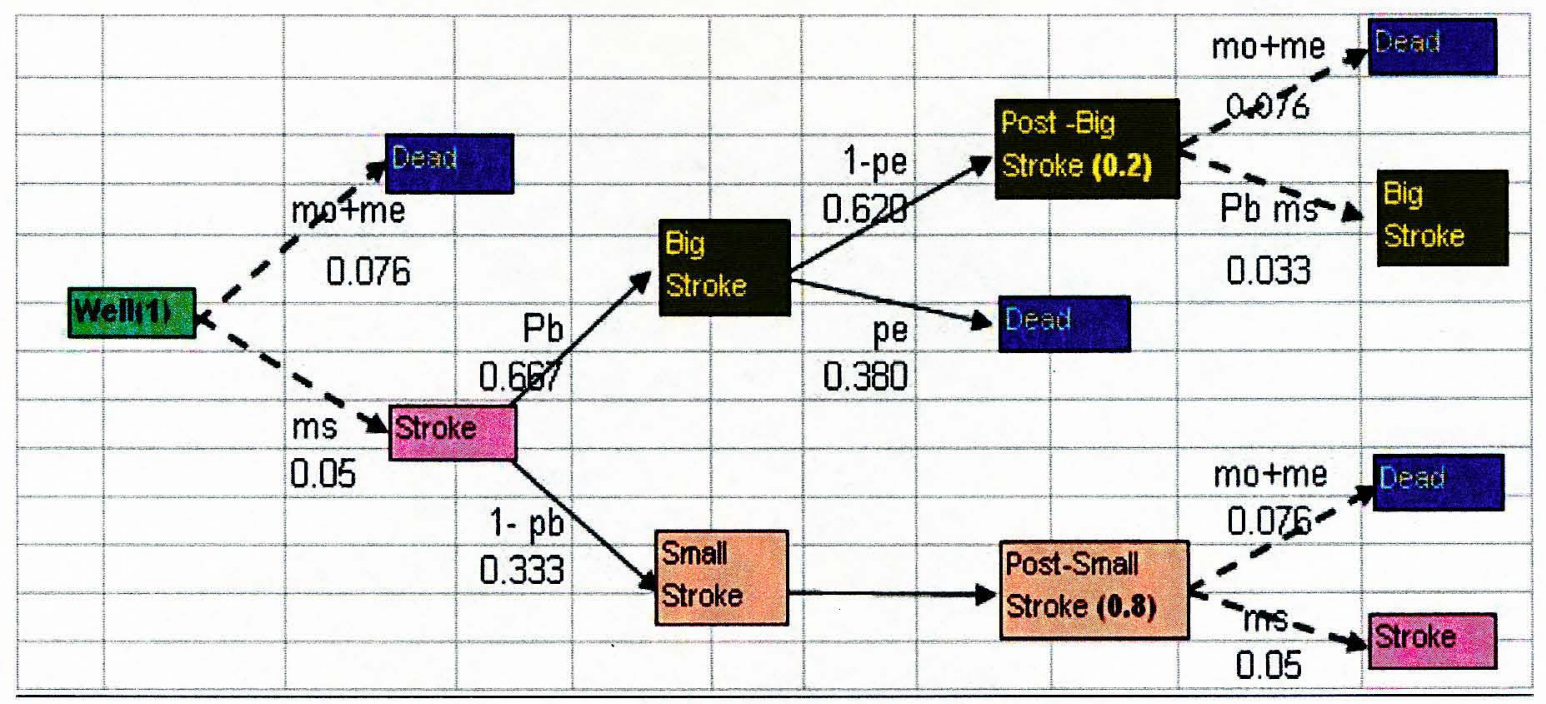

Figure 15: Stochastic Tree Model to Compute QALY for Stroke

Table 7: Results of Stochastic Tree Model to Compute QALY for Stroke

\begin{tabular}{|c|c|c|c|c|c|c|c|}
\hline $\mathrm{n}$ & Well & $\begin{array}{l}\text { Stroke } \\
\ll_{\text {Well }}\end{array}$ & $\begin{array}{l}\text { BigStroke } \\
\text { «stroke }\end{array}$ & $\begin{array}{l}\text { PostBigStr } \\
\ll_{\mathrm{Big}}\end{array}$ & $\begin{array}{l}\text { BigStroke } \\
\text { «postBig }\end{array}$ & $\begin{array}{l}\text { SmallStroke } \\
\text { «Stroke }\end{array}$ & $\begin{array}{l}\text { Stroke } \\
\text { «Small }\end{array}$ \\
\hline 0 & 0.0 & 0.0 & 0.0 & 0.0 & 0.0 & 0.0 & 0.0 \\
\hline 1 & 7.162 & 0.352 & 0.155 & 1.361 & 0.000 & 5.442 & 0.000 \\
\hline 2 & 7.163 & 0.353 & 0.163 & 1.432 & 0.105 & 5.445 & 0.003 \\
\hline 3 & 7.163 & 0.353 & 0.164 & 1.436 & 0.111 & 5.445 & 0.003 \\
\hline 4 & 7.163 & 0.353 & 0.164 & 1.436 & 0.111 & 5.445 & 0.003 \\
\hline
\end{tabular}

The symbol « means "reached by"

Table 1 shows that computing the QALY converges, because of Markov process, to the value of 7.163 years. To check the effect of Patient's conditions factor, on the Stroke example, we set Patient's Risk Assessment factor $\mathrm{r}(\mathrm{y})=0.12$ and three initial different values to Patient's conditions factor $\Phi=0.35,0.7,1.5$ and 3 , for WAFT. The QALY results: $8.128,7.947,7.608$, and 7.163 respectively.

We introduce a possible extension to the analytical solution, stochastic tree modeling. In specific, the Weibull accelerated failure time model was utilized instead of an exponential distribution. This enhancement may increase the stochastic tree modeling 
technique's capabilities, by using different state sojourn distribution and by including a correct boundary difference between experimental treatment and the standard of care.

However, the stochastic tree model utilizing the Weibull accelerated failure time model should be restricted to have a memory less property for the rollback computation with respect to the semi-Markov requirements. On other words, the WAFT model is used in Hazen's stochastic tree model framework, with an application to study stroke. The WAFT extends the stochastic tree model capabilities. In addition, inclusion of the WAFT in the stochastic tree model framework creates a correct boundary difference between experimental and the non-experimental regimens.

Further work would be an extension to the stochastic tree modeling technique by integration with simulation, and screening and selection procedures, to have more fully explore the response of stochastic tree models. To explore this response in a systematic way, multiple factors must be considered simultaneously with identifying "best" configurations combinations for some recurrent disease representation on stochastic tree.

The WAFT model is used in Hazen's stochastic tree model framework, with an application to study stroke. The WAFT extends the stochastic tree model capabilities. In addition, inclusion of the WAFT in the stochastic tree model framework creates a correct boundary difference between experimental and the non-experimental regimens.

A stochastic tree model using the WAFT model, by including a patient's health conditions, may more exactly calculate outcome measures associated with cyclic disorders or recurrent diseases/injuries. However, this new extended stochastic tree modeling technique still has a preference summary-memory less property. Future studies that combine simulations, screening and selection procedures, as well as multi attribute 
utility theory in the stochastic tree model framework are warranted to more fully explore the robustness of the stochastic tree model technique for recurrent diseases.

\subsection{Multiobjectives Simulation-Based Methodologies for Medical Decision Making}

We have applied our simulation model of treatment choice for ductal carcinoma in situ $(D C I S)$, a precancerous condition whose treatment is controversial (Hazen, Morrow and Venta 1999). Traditionally, DCIS was a rare disease treated by mastectomy, but modern mammography has converted this unusual entity into a common pathological finding (Silverstein et al. 1992, Hiramatsu et al. 1995). Recently the need for surgery as extensive as mastectomy has been questioned, and alternatives have been proposed such as lumpectomy, or lumpectomy in conjunction with radiation treatment or the drug tamoxifen. Here the possible interventions for the patient's ipsilateral breast are portrayed following diagnosis of DCIS. The possible interventions are mastectomy, lymphectomy followed by radiation therapy (XRT), or lumpectomy only. The data and the probabilities such as pSurgDeath (equal to $0.14 \%$ ) of surgical death under mastectomy are contained in a spreadsheet tables on the next two pages.

\section{Example:}

To utilize our simulation model to select the best DCIS treatment, the data tables required for the three treatment models are: transition probability matrix, transition rates matrix, quality of life table, initials for the simulation systems table, annual treatments cost table (synthetic data), and health states survival distribution table (synthetic data). 
Table 8

Quality of Life (QoL)

\begin{tabular}{|l|r|}
\hline State & QoL \\
\hline Death & 0.00 \\
Healthy & 1.00 \\
Immediate Cancer & 0.70 \\
Invasive Cancer & 0.70 \\
Post LymphOnly & 1.00 \\
Post LymphRad & 0.95 \\
Post Mastectomy & 0.90 \\
\hline
\end{tabular}

Table 10

Annual treatment Cost

\begin{tabular}{|lr|}
\hline State & Dollars \\
\hline Healthy & $\$ 500$ \\
Immediate Cancer & $\$ 7,000$ \\
Invasive Cancer & $\$ 5,000$ \\
Post LymphOnly & $\$ 6,000$ \\
Post LymphRad & $\$ 4,000$ \\
Post Mastectomy & $\$ 3,000$ \\
\hline
\end{tabular}

values are synthetic
Table 9

Health State's Stay Time Distributions, in years

\begin{tabular}{|ll|}
\hline State & Survival distributions \\
\hline Death & 0 \\
Healthy & Norm(35,5) \\
Immediate Cancer & Weib $(0.15,0.5)$ \\
Invasive Cancer & Epxo(0.08) \\
Post LymphOnly & Weib $(0.00087,3.0)$ \\
Post LymphRad & Cont $(0,0, .25,4, .50,9, .75,11,1,27)$ \\
Post Mastectomy & Norm $(7,5)$ \\
\hline
\end{tabular}

values are synthetic

Table 11

Initial Values for the simulation system

\begin{tabular}{|l|c|}
\hline Variable & Initial Value \\
\hline Patient's Risk Factor & 0.10 \\
Decision Maker's Risk Factor & 0.90 \\
Cost Discount Factor & 0.01 \\
W1 & 0.60 \\
W2 & 0.40 \\
State of Life & changeable due to model \\
\hline
\end{tabular}


Table 12

Transition Probability Matrix

\begin{tabular}{|l|ccccccc|}
\hline State & Death & Healthy & $\begin{array}{c}\text { Immediate } \\
\text { Cancer }\end{array}$ & $\begin{array}{c}\text { Invasive } \\
\text { Cancer }\end{array}$ & $\begin{array}{c}\text { Post } \\
\text { LymphOnly }\end{array}$ & $\begin{array}{c}\text { Post } \\
\text { LymphRad }\end{array}$ & $\begin{array}{c}\text { Post } \\
\text { Mastectomy }\end{array}$ \\
\hline Death & 0 & 0 & 0 & 0 & 0 & 0 & 0 \\
Healthy & 0 & 0 & 0 & 0.39 & 0 & 0.61 & 0 \\
Immediate Cancer & 1.00 & 0 & 0 & 0 & 0 & 0 & 0 \\
Invasive Cancer & 1.00 & 0 & 0 & 0 & 0 & 0 & 0 \\
Post LymphOnly & 0 & 0.98 & 0.02 & 0 & 0 & 0 & 0 \\
Post LymphRad & 0 & 0.98 & 0.02 & 0 & 0 & 0 & 0 \\
Post Mastectomy & 0 & 0.98 & 0.02 & 0 & 0 & 0 & 0 \\
\hline
\end{tabular}

\section{Table 13}

Transition Rates

\begin{tabular}{|l|ccccccc|}
\hline State & Death & Healthy & $\begin{array}{c}\text { Immediate } \\
\text { Cancer }\end{array}$ & $\begin{array}{c}\text { Invasive } \\
\text { Cancer }\end{array}$ & $\begin{array}{c}\text { Post } \\
\text { LymphOnly }\end{array}$ & $\begin{array}{c}\text { Post } \\
\text { LymphRad }\end{array}$ & $\begin{array}{c}\text { Post } \\
\text { Mastectomy }\end{array}$ \\
\hline Death & 0 & 0 & 0 & 0 & 0 & 0 & 0 \\
Healthy & 0 & 0 & 0 & 0.044 & 0 & 0 & 0 \\
Immediate Cancer & 0.001 & 0 & 0 & 0 & 0 & 0 & 0 \\
Invasive Cancer & 0.003 & 0 & 0 & 0 & 0 & 0 & 0 \\
Post LymphhOnly & 0 & 0 & 0 & 0 & 0 & 0 & 0 \\
Post LymphRad & 0 & 0 & 0 & 0 & 0 & 0 & 0 \\
Post Mastectomy & 0 & 0 & 0 & 0 & 0 & 0 & 0 \\
\hline
\end{tabular}

in case of Lymphh node treatment model, the value 0.044 be 0.017 


\subsubsection{The Simulation Model on the Stochastic Tree to Compute the Expected Utility}

function of QALY and Cost attributes of three Breast Cancer Treatments

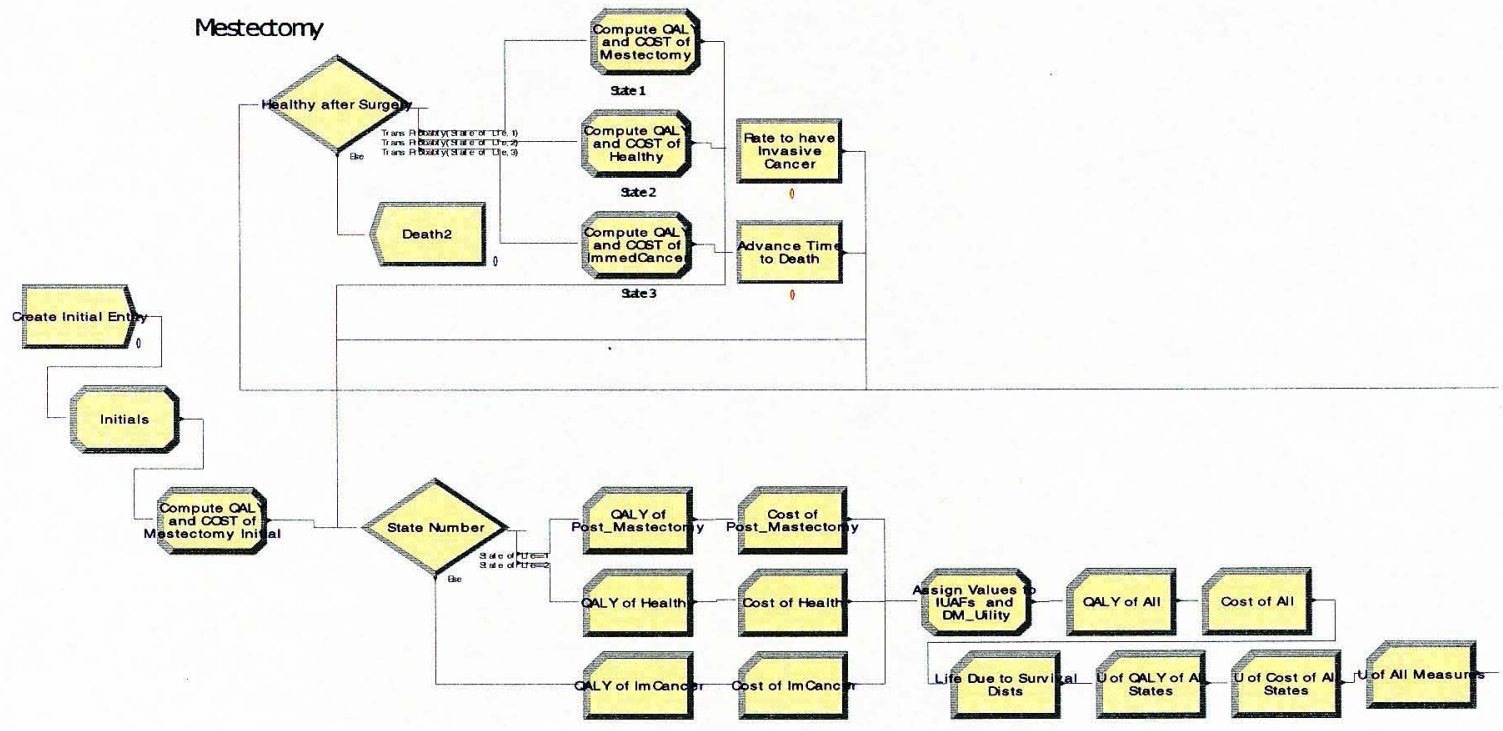

Figure 16: The Simulation model for Mastectomy

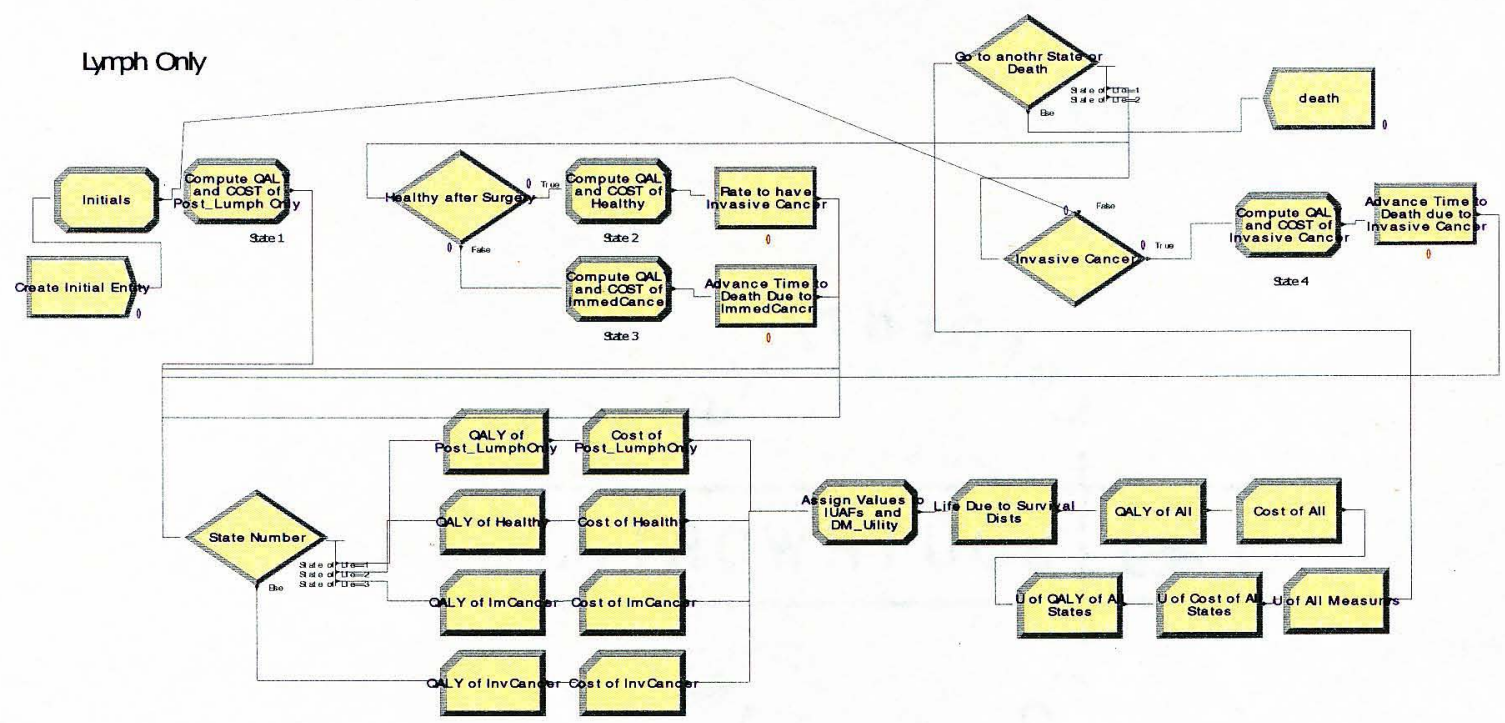

Figure 17: The Simulation model for Lumpectomy Only 


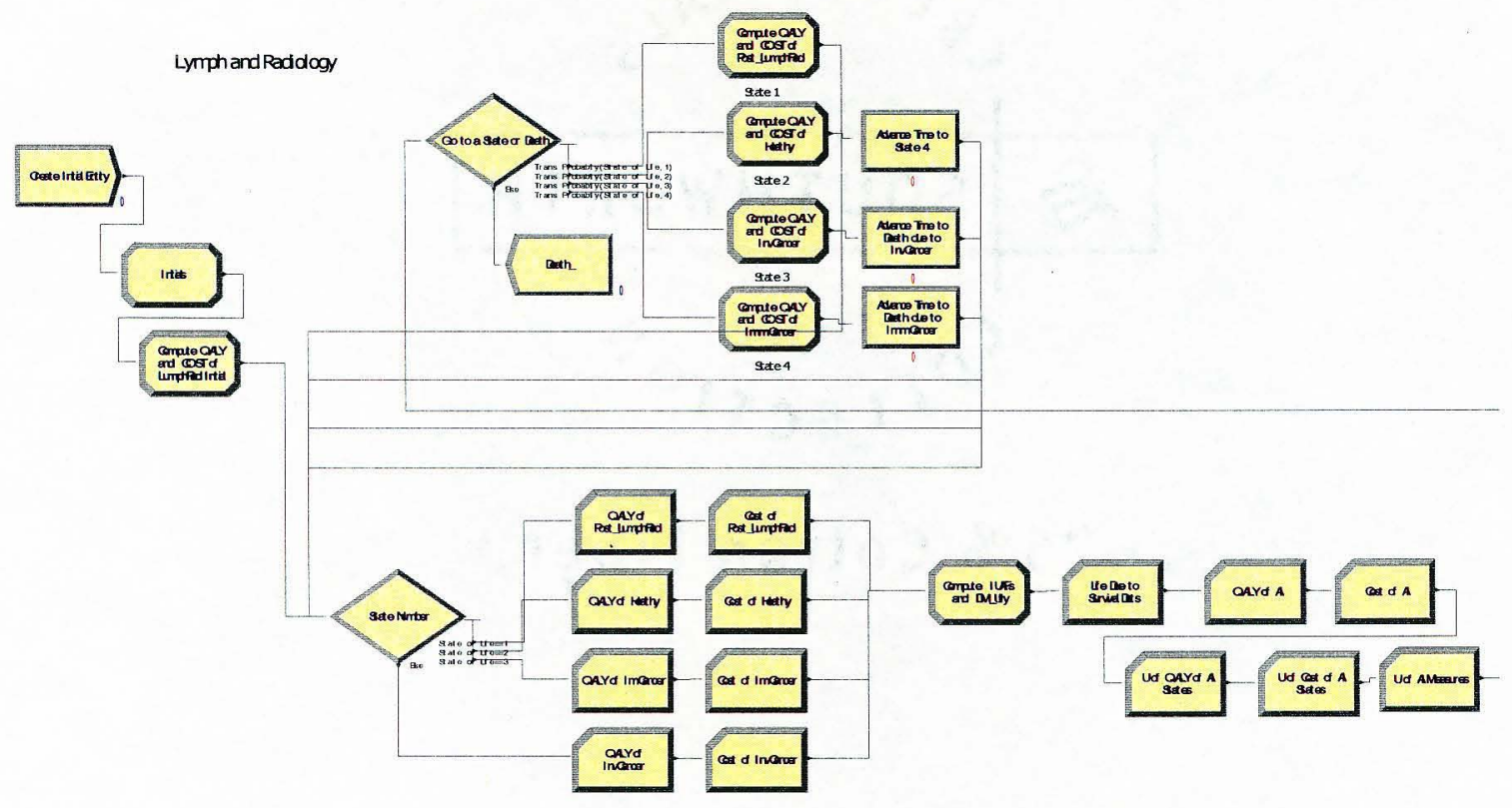

Figure 18: The Simulation model for Lumpectomy followed by Radiation therapy

\subsubsection{Results of our Simulation Model on the Stochastic Tree}

For the three DCIS treatments three simulation systems developed, $k=3$, the above data tables utilized, two utility dependent performance measures, QALY and Cost, included, the patient's risk assessment factor set to $10 \%$, decision maker's risk assessment factor set to $90 \%$, initial replications $b_{o}$ set to 20 , and the significant difference $\delta$ set to 0.006. Using Dudewicz and Dalal (1975) or Rinott, (1978): the firststage sample means and sample variances, $S_{i}$ were computed. The sample variances are used to determine the number of macro-replications which must be taken in the second stage

$$
b_{i}=\max \left\{b_{0},\left[\left(\frac{h S_{i}}{\delta}\right)^{2}\right]\right\}
$$


$h$ is a function in the number of replications $b_{0}$, the number of systems $k$, and $\delta$. the value of $h$ was determined ( $h=2.431$ ), see Bechhofer, (1995), page 62.

For $b_{0}$ replications, we computed grand means $\bar{Y}_{i}=\sum_{j=1}^{b_{i}} \frac{\bar{Y}_{i j}}{b_{i}}, i=1,2, \ldots, k$ and selected the system with largest $\bar{Y}_{i}$

The macro-replication estimators, $\overline{Y_{i 1}}, \overline{Y_{i 2}}, \ldots, \overline{Y_{i b_{i}}}$ from the $\mathrm{i}^{\text {th }}$ system are assumed to be i.i.d. with expectation. If the number of micro-replications is large enough the Central Limit Theorem (CLT) yields approximate normality for the macro-replication estimators.

Table 14: CDIS pilot experiment; the first stage results

\begin{tabular}{|c|c|c|c|}
\hline $\mathrm{i}$ & L\&Rad & L.only & Mastec \\
\hline$\left.y^{(}\right)$ & 0.485 & 0.442 & 0.492 \\
\hline$S_{i}$ & 0.01536 & 0.0108 & 0.02402 \\
\hline$s_{i}$, & 0.0034 & 0.0024 & 0.0054 \\
\hline$b_{i}$ & 38 & 20 & 94 \\
\hline
\end{tabular}

In stage two, to compute the grand means $\bar{Y}_{i}$, we re-run the simulation model of Lumpectomy followed by Radiation therapy (L\&Rad) 38 macro-replications, and we rerun the simulation model of Mastectomy 94 macro-replications. The results:

Table 15: CDIS the second stage results

\begin{tabular}{|c|c|c|c|}
\hline $\mathrm{i}$ & L\&Rad & L.only & Mastec \\
\hline$Y_{i}^{(1)}$ & 0.485 & 0.442 & $\mathbf{0 . 4 8 7}$ \\
\hline$s_{i}$ & 0.0024 & 0.0024 & 0.0029 \\
\hline
\end{tabular}


We are at least $90 \%$ sure that we have made the correct selection $\left(u_{(1)}-u_{(2)}>\delta=0.006\right)$; The best system will be the one with the max average; which is Mastectomy.

\subsubsection{Sensitivity Analysis}

A sensitivity analysis was made to check whether selecting Mastectomy was sensitive to the change of patient's and decision maker's risk assessment factors. The patient's risk assessment factor changed from $10 \%$ to $90 \%$, and the decision maker's risk factor changed from $90 \%$ to $10 \%$. For the same three systems of DCIS treatment and for initial replications $b o=20$, the pilot experiment of the first stage is shown on table \#\#

Table 16: CDIS pilot experiment for the sensitivity analysis; the first stage results

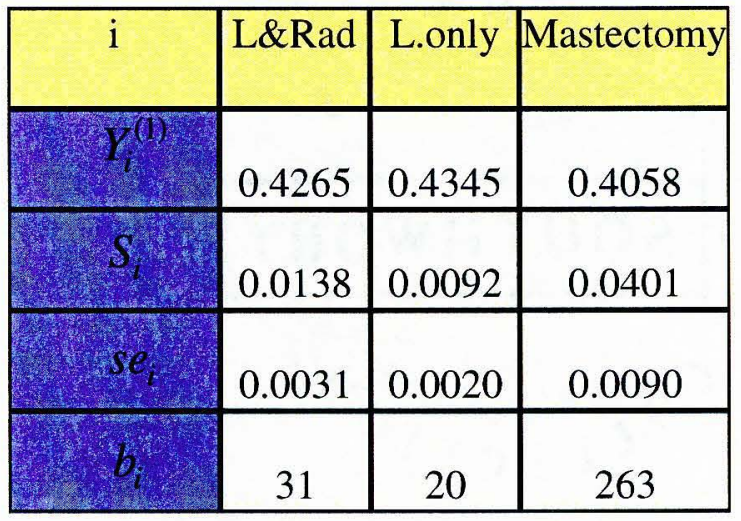

For the final results of the pilot experiment after changing the patient's and the DM's risk assessment factors, in stage two, we re-run the L\&Rad model 31 macro-replications; and we re-run the mastectomy model 263 macro-replications.

Table 17: CDIS the second stage results, for the sensitivity analysis

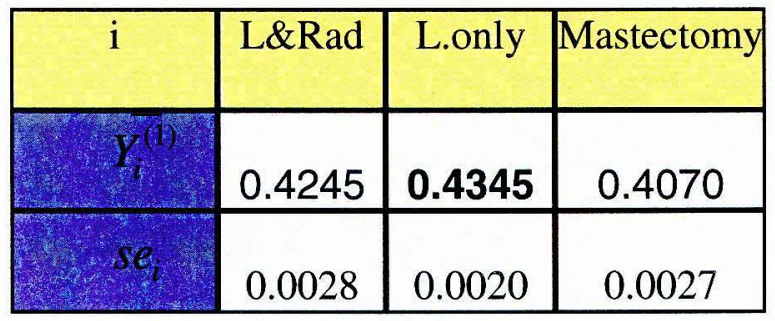


The results show that the decision was sensitive to the change of patient's risk factor and the change of DM's risk factor, and the best system is changed to be lumpectomy only instead of Mastectomy.

To check the effect of patient's illness conditions factor, utilizing Weibull Accelerated Failure Time model, on the expected utility of lumpectomy followed by radiation therapy of DCIS, we run the Lymph and Radiation simulation model (figure \#) with different stay time distributions. We set the stay time of the Immediate Cancer and Invasive Cancer health states to be weibull $(\Phi \lambda, 1)$, where $\lambda$ is the scale parameter $(0.08)$, and $\Phi$ is the patient's illness conditions factor, $\Phi=1,2,3,4$, and 5 .

For $n=30$ replications, patient's risk assessment factor 0.50 , decision maker' risk assessment 0.50, and for two utility dependent performance measures, QALY and Cost, the results is shown on the following figure 19

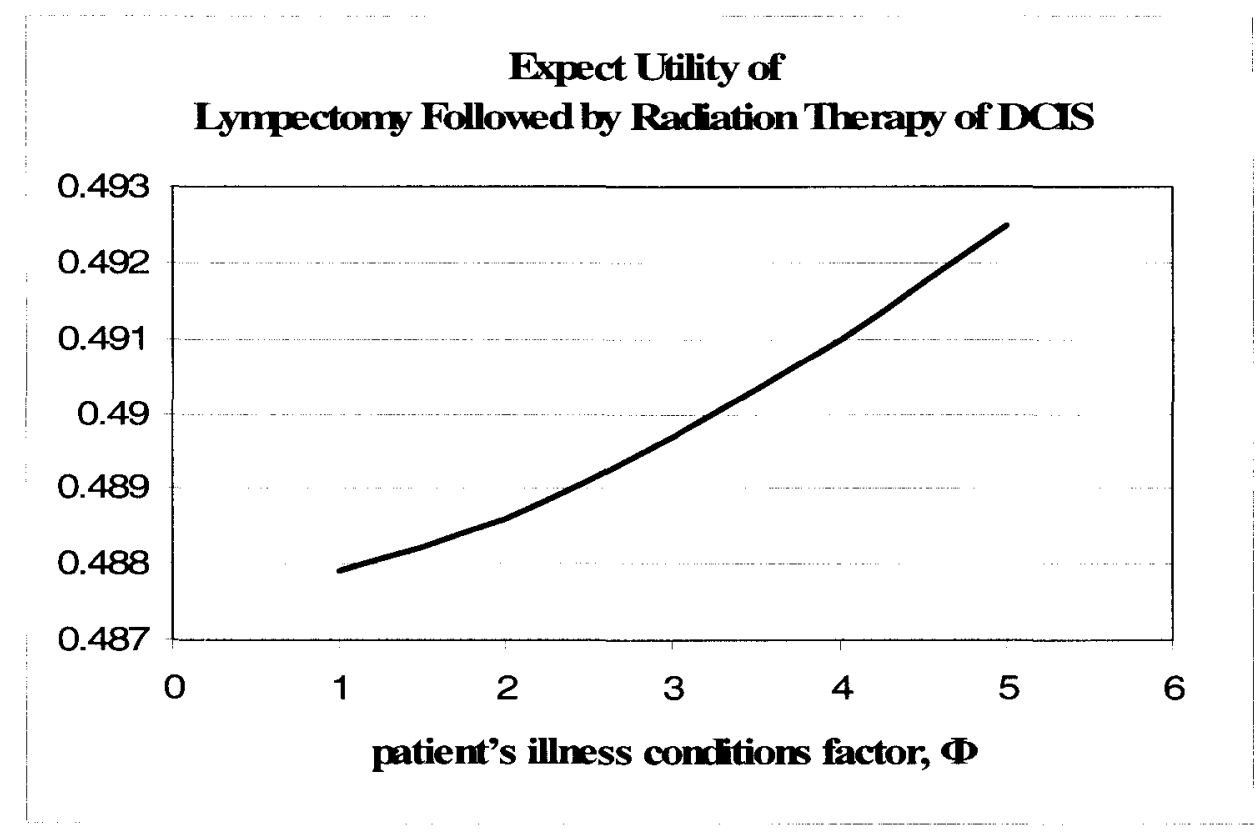

Figure 19: Expected utility of Lymph Radiation Therapy. The curve shows that the expected utility changes when the patient's illness conditions factor changes. 


\subsection{Discussion and Conclusion}

The importance of Hazen's stochastic tree was mentioned in the technical report, by Prof. Keefer et al (2002), "Summary of Decision Analysis Applications in the Operations Research Literature, 1990-2001". The report declared that the work of Hazen et al. (1998), who provided an introduction to stochastic trees and related software to be used in medical decision making, is one of the application that presented significant detail about a particular decision analysis methodological issue (Keefer, 2002). However, there are still no widely accepted techniques for incorporating variability in the estimates of the parameters/ performance measures of a decision tree.

For complex stochastic decision trees with random variable parameters, simulation application of such a system is important in order to introduce key components and processes that provide a source of data for reliable implementation. One of the many problems for which simulation has been found to be a useful and powerful tool is designing and operating stochastic models, such as probabilistic trees. The stochastic tree is a model which could integrate of simulations, survival analysis approaches, and ranking-selection procedures (Hazen, 1992, Hazen \& Pellissier, 1996). It would be beneficial to add development to the stochastic tree for decision analyses for medical intervention in recurrent diseases.

In this research, we used parametric survival models, such as the Weibull Accelerated Failure Time Model, to include patient's conditional survival probability accurately. Moreover, in our enhanced model on Hazen's stochastic tree, we combined the Weibull Accelerated Failure Time model into our model to fill the gap between the 
experimental and actual/current medical intervention for recurrent diseases or injury. Also, our multi-objective simulation-based model has the facility to use not only exponential distribution for states sojourn but also the parametric survival models too.

"There have been fewer methods proposed and thus little software developed for analyzing multiple test data, due to difficulties relating to the covariances among the variables" Westfall et al. (1999). Our multi-objective simulation-based model developed for analyzing multiple performance measure/test without difficulties relating to the covariances among the variables.

Our multi-objective simulation-based model gives the facility to the decision maker to set his/her policy through the use of Multi-Attributes Utility theory (MAU). Moreover, the use of MAU is appropriate for multi-objective functions; this would be an advantage over the dynamic programming principle. In the analysis, utility theory involves gathering the measures into a "Unified-Measure" of the desirability of each alternative. Utility theory provides the basis for the appropriate approach to combine the apparently unlike measures.

Our simulation model is easy to use and compute in chronic cases, when the chance of moving between health states increases with age. It fits with the fact that aging increases the risk of transition from a healthy state to one of sickness, thereby violating the constant state-transition probability assumption in Markov chains (e.g. using the Weibull Accelerated Failure Time Model). Our system can behave as Markov processes that are used to model disease over longer time periods. In addition to behaving similarly to the pure Markov model our simulation model fits with the semi-Markov process model. It is a model useful in analyzing complex dynamic systems, and its transition rates 
in a particular state depend on the time already spent in that state (sojourn time), but they do not depend on the path by which the present state was reached.

In our simulation model, we used the MAU to unify multi tests (multiperformance measures) into one scale (Morrice, 1998). Thus we utilized a RankingSelection procedure of Rinott, (1978), to select the system with the largest expected performance measures (Bechhofer 1995, Kim-Nelson 2001, Nelson-Miler 1995).

The weights represent another confounder and affect the relative importance of a performance measures, its impact on the variation, and its impact on the computing should be tested by sensitivity analysis. Our example, of three medical interventions for breast cancer treatment, demonstrates that our approach can be applied to realistic medical problems in which simulation is utilized.

The idea presented by Chick et al $(1998,2001)$, who use a Bayesian technique to determine the number of additional replications required to reduce the risk of an incorrect selection, one could extend our simulation based methodology to include their work; however, their work focuses on a single criterion. That would ease some of the computational burden associated with the used R\&S procedures. Using of common random numbers would facilitate variance reduction to our work that has combination with R\&S procedures (Nelson et al 1995 and Goldsman et al 1998). 


\subsection{Our approach Advantages.}

Applying R\&S procedures on MAU theory outputs allows good performance on one criterion to compensate for poor performance on another, and we assess the comparative importance of each criterion. Previous work on multivariate $R \& S$ procedures has been limited by the lack of a trade-off method that allows the decision maker to combine explicitly different performance measures (Gupta, 1979).

Our approach closely relates to the common medical-business management of assigning preferences to different performance measures. Similar to the solution achieved by Morrice et al (1998 and 2001), in industry, our solution does not require complications like a covariance matrix, which was used by Gupta (1979).

Our approach leads to a reasonable decision; for example, it avoids Kim and Lin (1999) approach that in some situations focuses on maximizing the poorest performing criterion (they used the MaxiMin approach). And, unlike Kim and Lin, we do not perform "several approaches for the final decision"

Certainly, there are several issues that must be recognized as important for the success of our approach. Because we use a pay off model this requires elicitation by decision maker of both a utility function for each measure, for example: QALY and COST, as well as a "weight," or indication of relative importance. Techniques for handling these types of elicitations are available (e.g. Clemen 1991 and Keeney and Raiffa 1976). And for patient's utility assessment, we use Hazen's pay off approach; this requires the physician to elicit from the patient his or her risk assessment.

The idea for the methodology outlined in this research is based on integration of an enhanced medical stochastic model, for evaluating multi medical 
intervention/treatment outcomes, with real decision makers, who use decision inputs computed by simulation experiments combining patient's preferences, condition, and health states.

Our work result represents a combination of two sound theories: multi-attribute utility theory and ranking and selection with an enhancement/extension of Hazen's stochastic tree model using simulation. This synthesis extends the use of medical stochastic models, simulation, and ranking-selection literature by accommodating multiple criteria, and provides another application area for multi-attribute utility theory. 


\section{REFERENCES}

- Banks, (1995): Jerry Banks, John S. Carson II, Barry L. Nelson, Discrete-Event System Simulation, Prentice Hall.

- Bechhofer, (1995): Bechhofer, R. E., T. J. Santner, and D. Goldsman. Design and Analysis of Experiments for Statistical Selection, Screening and Multiple Comparisons. New York: John Wiley and Sons.

- Bryg , (1994): David J. Bryg, Comparison of Nevada simulation to Monte Carlo simulation Winter Simulation Conference, Proceedings of the 26th conference on Winter simulation, $471-476$.

- Butler, (1997): Butler, J.C., J. Jia, J.S. Dyer. Simulation techniques for the sensitivity, analysis of multi-criteria decision models. Eur. J. Oper. Res. 103 531-545.

- Chamber, (2002): Michael G. Chamber, Peter Koch, Hutton; Development of a Decision-Analytic Model of Stroke Care in the United States and Europe; Value in Health, vol.5 number 2.

- Chen (2000), Chen, H. C., C. H. Chen, and E. Yücesan. Computing efforts allocation for ordinal optimization and discrete event simulation. IEEE Transactions on Automatic Control 45:960-964

- Chick (2001), Chick, S., and K. Inoue. New two-stage and sequential procedures for selecting the best simulated system. Operations Research 49:1609-1624. 
- Clarke, (2004): Sally-Ann Clarke, Christine Eiser; The measurement of health-related quality of life QOL in Paediatric clinical trials: A Systematic Review; Health and Quality of Life Outcomes, 2:66 doi: 10.1186/1477-7525-2-66.

- Clemen, (1990): Robert T. Clemen, Making Hard Decisions, by Duxbury, ISBN 0$534-92336-4$

- Collett , (1999): D. Collett, Modeling Survival Data in Medical Research, Texts in Statistical Sience, Publisher: Chapman and Hall/CRC, UK.

- Detsky, (1996): Allan S. Detsky et al, Decision Analysis, SMDM; Dec. 12.

- Detsky, (1997): Allan S. Detsky, et al, Building a Tree, MDM; 17: 126-135

- Dittus, (1990): Dittus, R. S., R. D. Eisenhut, R. W. Klein, S. D. Roberts, M. S. Stouppe, and J. R. Wilson. TreeModeler: Construction and analysis of probability trees with random variable parameters. Research Memorandum 90-1, School of Industrial Engineering, Purdue University, West Lafayette, Indiana.

- Drummond, (1997): Drummond, MF, O'Brien, B, Stoddart, GL, and Torrance, GW. Methods for the economic evaluation of health care programs. Second ed. Oxford: Oxford University Press.

- Dyer, (1998): Dyer, J.S., T. Edmunds, J.C. Butler, J. Jia.. A multiattribute utility analysis of alternatives for the disposition of surplus weapons-grade plutonium. Oper. Res. 46(6) 749-762.

- Feinberg , (2002): Feinberg and Shwartz, Handbook of MDP- Methods and Applications, Kluwer's International Series.

- Feinberg, (2002): Eugene A. Feinberg, Adam Shwartz, Handbook of Markov Decision Processes Methods and Applications, by Kluwer's International Series. 
- French, (2000): S. French, and D. Rios Insua, Statistical Decision Theory, Kendall's Library of Statistics, book by Arnold, a member of Hodder Headline Group, London, ISBN 0-340-61460-9.

- Green, (2003): Peter Green, Nils Hjort, and Sylvia Richardson, Highly structured stochastic systems book, by Oxford statistical science series.

- Gupta, (1979): Shanti S. Gupta, S. Panchapakesan, Multiple Decision ProceduresTheory and Methodology of Selectin and Panking populations, book, by Wiley Series in Probability and Mathematical Statistics.

- Hazen, (1992): Gordon B. Hazen, Stochastic Trees: A new technique for temporal medical decision modeling. Medical Decision Making 12, 163-178.

- Hazen, (1996): Gordon B. Hazen, James M. Pellissier, Recursive Utility for Stochastic Trees, Operations Research, Vol. 44 No. Sep.-Oct.

- Hazen, (1996 b): Gordon B. Hazen, James M. Pellissier, Rowland W. Chang, Continuous-risk Decision Analysis of Total Hip Replacement, journal of the operational Research Society 47, 776-793.

- Hazen, (1998): Gordon B. Hazen, James M. Pellissier, Jayavel Sounderpandian, Stochatic-Tree Models in Medical Decision Making, Interface 28: pp. 64-80 4 JulyAugust.

- Hazen, (1999): Gordon B. Hazen and J. Sounderpandian, Preference Summaries for Stochastic Tree Rollback. In Beliefs, Interactions and Preferences in Decision Making, Netherlands. pp. 109. 
- Hazen, (2002): Gordon B. Hazen, et al, Stochastic Trees and the Stotree modeling environment: models and software for medical decision analysis, J. Med. Systems; $26399-413$.

- Heyman, (1990): D.P.Heyman M.J.Sobel, Stochastic Models: Handbooks in Operation Research and Management Science, North_Holand.

- Jaafari, (2003): Ali Jaafari, Junkui Yao, Combining real options and decision tree: An integrative approach for project investment decisions and risk management, Journal of Structured and Project Finance. New York: Vol.9, Iss.3; pg.53

- Keefer, (2002): Donald Keefer et al, Arizona State University,, Technical Report, Summary of Decision Analysis Applications in the Operations Research Literature, 1990-2001, in Nov.

- Kelton, (2001): David Kelton et al, Simulation with Arena.

- Kim, (2003): Kim, S.-H. and B. L. Nelson. Selecting the best system: Theory and methods. Proceedings of the 2003 Winter Simulation Conference. S. Chick, P. J. Sanchez, D. Ferrin and D. J. Morrice, eds. 101-112. New Orleans, Louisiana: Institute of Electrical and Electronics Engineers

- Law, (2000): Law, A. M., and W. D. Kelton. Simulation modeling and analysis, 3d ed. New York: McGraw-Hill.

- Moore, (2001): Moore, Decision Modeling with Microsoft Excel, 6th edition, Prentice Hall on.

- Morrice, (1998): Douglas J. Morrice, John Butler, and Peter W. Mularkey. An Approach to Rank and Selection for Multiple Performance Measures, Proceedings of the 1998 Winter Simulation Conference. 
- Morrice, (1999): Morrice, D.J., J.C. Butler, P.W. Mullarkey, , S. Gavirneni.

Sensitivity analysis in ranking and selection for multiple performance measures. P.A. Farrington, H.B. Nembhard, D.T. Sturrock, G.W. Evans, eds. Proc. 1999 Winter Simulation Conf. The Institute of Electrical and Electronic Engineers, Piscataway, NJ., 618-624.

- Morrice, (2001): Douglas Morrice, John Butler, Peter W. Mullarkey, A Multiple Attribute Utility Theory Approach to Ranking and Selection, Management Science, 2001 Informs, Vol 47, No.6, June 01 pp. 800-816.

- Nelson, (1998): Goldsman, D., and B. L. Nelson. Comparing systems via simulation. In Handbook of Simulation, ed. J.Banks, 273-306. New York: John Wiley.

- Nelson, (2001): Nelson, B. L., J. Swann, D. Goldsman, and W.-M. T. Song. Simple procedures for selecting the best system when the number of alternatives is large. Operations Research 49:950-963.

- Parlar, (1990); Mahmut Parlar; Stochastic Decision Tree Analysis on an Electronic Spreadsheet, Computers and Engng Vol. 18, No 2, pp 255-234.

- Pliskin, (1988): Pliskin, Incorporating Stochastic Processes to Overcome Unmanageable Decision Trees; European Journal of Oper. Research; Vol. 34, Iss. 2; pg. 149.

- Raiffa, (1992) Raiffa, H. Decision Analysis: Introductory Lectures on Choice Under Uncertainty, Addison-Wesley.

- Rinott, (1978): Yosef Rinott. on Two-Stage Selection Procedures and Related Probability-Ineqalities, Commu. Statist-Theor. Meth., A7(8), 799-811. 
- Schoemaker, (1982): Schoemaker, P. J. and C. C. Waid. An Experimental Comparison Of Different Approaches to Determining Weights in Additive Utility Models. Management Science, 28:182-196.

- Westfall, (1999): Westfall, P.H., Tobias, R.D., Rom, D., Wolfinger, R.D. and Hochberg, Y. Multiple Comparisons and Multiple Tests using the SAS System, SAS Books by Users, to appear. 


\section{CURRICULUM VITAE}

NAME:

ADDRESS:

DOB:

\section{EDUCATION}

\& TRAINING:
Ahmed Youssef-Agha

The School of Public Health, The University of Louisville, Louisville, KY.

Egypt, Mar 8, 2006

B.Sc, Mathematics \& Education. The University of Menofia, Egypt 1981-1985

MSCS, Computer Sciences, The University of Louisville, $\mathrm{KY}$ 1999-2001

MSPH. Biostatistics-Decision Science, The University of Louisville, $\mathrm{KY}$ 2001-2003

PhD, Biostatistics-Decision Science. The University of Louisville, $\mathrm{KY}$ 2003-2006

AWARDS: "Teaching Honor ship Roll", 1999, from the American University in Cairo, Egypt

\section{COMPUTER SKILLS:}

Statistical Packages: SAS, SPSS, and R; Programming Languages: Delphi Mat Lab. 1 and Visual Basic; Database Management: SQL, FoxPro, and Microsoft Access: Miscellaneous: MS-Office, 'TreeAge, and AREANA

\section{PROFESSIONAL MEMBERSHIP}

IELE (omputer Society; American Statistical Association; INFORMS; and Sockiz of Medical Decision Making 


\section{REPORTS / PUBLICATIONS}

Ahmed YoussefAgha MSCS, MSPH, Steven McCabe, MD, MSPH, Gerald Evans, PhD. "Multiobjective Simulation-Based Methodologies for Disease/Injury Treatment", Abstract, Health Applications Section, INFORMS Annual Meeting- - 2005- San Francisco.

Chao,C., Studts,J.L., Abell,T., Hadley,T., Roetzer,L., Dineen,S., Lorenz,D., YoussefAgha,A., \& McMasters,K.M. Adjuvant Chemotherapy for Breast Cancer: How Presentation of Recurrence Risk Influences Decision-Making. J Clin.Oncol., 21(23): 4299-4305. <PM:14581440> (2003).

Ahmed YoussefAgha MSCS, MSPH, Steven McCabe, MD, MSPH, John Myers, Ph.D. Gerald Evans, PhD, Adel El-Maghraby, Ph.D.; "An Extension of the Stochastic Tree Model Using the Weibull Accelerated Failure Time Model" Submitted for publication at Medical Decision Making.

Ahmed YoussefAgha MSCS, MSPH, Steven McCabe, MD, MSPH, Resad Pasic, MD, Ph.D., Gerald Evans, PhD. "The Influence of Decision Sharing on Outcome Measures Following a Hysterectomy" Submitted for publication at Medical Decision Making.

Ahmed YoussefAgha, "Redesigning UofL Occupational Health Surveillance Program Database: a Technical Report", Submitted to the department of Environmental \& Occupational Health Science, the School of Public Health, the University of Louisville, $\mathrm{KY}$.

Ahmed YoussefAgha, "The Private Sector Colleges in Computer Science: A Research Report to Maintain Competitive Positioning in Computer Education Marketplace", Submitted to the Computer Studies Division, the Center of Continuing Education, the American University in Cairo, 1999.

Ahmed YoussefAgha and Amera Mekky, "The Egyptian Industries' Needs for Entering the International Markets: MIS Outputs and Economical/Statistical Analyses Report" Submitted to the Ministry of International Cooperation, Egypt, 1996.

Ahmed YoussefAgha and Shyrrein Bassam, "Old Cairo Tanneries Relocation Project for the Ministry of International Cooperation: MIS Outputs and Economical/Statistical Analyses Report" Submitted to the Egyptian Leather Industry Chamber, Egypt, 1995.

Ahmed YoussefAgha and Yasmien Jurdon, "Zanussi and Electrolux Household appliances: A Statistical Analyses Report" Submitted to the IDC for the two private sector companies of Egypt, 1995. 
Ahmed YoussefAgha and Dr. Marcello Giugale "The Implications of Regulations for Small Enterprises in Egypt: A Economical/Statistical Analyses Report" Submitted to the IDC for the Ford Foundations, 1994.

Ahmed YoussefAgha and Hussien Morrad, "Pilot Business Incubation Center Program for Social Fund for Development: A feasibility study Report" Submitted to the IDC for establishing two pilot business "incubators" in two cities in Egypt, 1993.

Ahmed YoussefAgha and Souzy Kammel, "Egyptian Private Sector Company (Confidential), Car Production Project: A Market Assessment Report" Submitted to the IDC, 1992.

Ahmed YoussefAgha, "Training Block Grants Information System: An MIS to Monitor and Report on Implementation of the Training Block Grant activity in 26 states of Egypt" Submitted to the IDC for the U.S. Agency for International Development, in Egypt, 1990. 